\title{
Nine new species of Thottea (Aristolochiaceae) in Peninsular Malaysia and Singapore, with two taxa in Peninsular Malaysia redefined and a taxon lectotypified
}

\author{
T.L. Yao ${ }^{1}$
}

\section{Key words}

conservation status endemic

new species

Peninsular Malaysia

Singapore

Thottea

\begin{abstract}
Nine new species of the genus Thottea, namely $T$. anthonysamyi, T. kamarudiniana, T. longipedunculata T. papilionis, T. piscodora, T. reflexa, T. ruthiae and T. terengganuensis from Peninsular Malaysia (eight species) and T. praetermissa from Peninsular Malaysia and Singapore (one species) are described and illustrated. Distribution maps are provided and conservation status is assessed for the new species. Thottea dependens and $T$. tricornis are redefined and T. parviflora is lectotypified. Thottea is now represented by 16 species in Peninsular Malaysia and two in Singapore. A spherical-ovoid shaped perianth with an aperture at the top is observed for the first time in Thottea. The white and bicoloured perianth are described for the first time in Peninsular Malaysian Thottea. Eight out of the nine novelties fall in the IUCN 'Threatened' category, and six of them are considered as 'Critically Endangered'.
\end{abstract}

Published on 21 November 2013

\section{INTRODUCTION}

Thottea Rottb. (Aristolochiaceae) is a small genus with about 35 species distributed in Sri Lanka, India, Bangladesh, Myanmar, Thailand, Vietnam, China (Hainan), Sumatra, Peninsular Malaysia, Singapore, Java, Borneo, the Philippines and Sulawesi. In Malesia, the taxonomic study of Thottea reached the highest achievement in the publication of the Aristolochiaceae account in Flora Malesiana (Hou 1984), in which 22 species are recognised, 7 of which occur in Peninsular Malaysia, namely Thottea dependens (Planch.) Klotzsch, T. grandiflora Rottb., T. parviflora Ridl., T. piperiformis (Griff.) Mabb., T. sumatrana (Merr.) Ding Hou, T. tomentosa (Blume) Ding Hou and T. tricornis Maingay ex Hook.f.; and two in Singapore, namely $T$. dependens and T. grandiflora. All four Thottea species that are found in Thailand, T. grandiflora, T. parviflora, T. sumatrana and T. tomentosa (Phuphathanaphong 1987) also occur in Peninsular Malaysia.

Morphologically similar species are difficult to distinguish during non-reproductive stages. Flowering of some Thottea species is intermittently seasonal (Kiew 1984, pers. obs.) and thus flowering specimens are not always available. Inflorescence position, shape, size and colour of perianth, as well as stamen number and arrangement, provide very important characters for species identification (Shaiju \& Omanakumari 2009, 2010). Ultrastructural features of the abaxial surface of the lamina also facilitate species identification of non-reproductive specimens (Hou 1981) but these characters are only useful for some taxa. Recent phylogenetic analyses demonstrate the monophyly of Thottea (Oelschlägel et al. 2011).

\section{Discovery of Thottea in Peninsular Malaysia and Singapore}

The history of Thottea-discovery in Peninsular Malaysia mirrors that of taxonomic research in Malay Peninsula (Kiew et al. 2010). All taxa from the Malay Peninsula treated by Hou (1984), except $T$. sumatrana, were discovered and named

\footnotetext{
${ }^{1}$ Forest Research Institute Malaysia, 52109 Kepong, Selangor, Malaysia; e-mail: yaotzeleong@frim.gov.my.
}

before the publication of Ridley's Flora of Malay Peninsulain the 1920s. These species occur mainly in the west coast of Peninsular Malaysia.

After an interval of about 50 years, Thottea longipedunculata was collected by a forester A. Motan (KEP) in 1960. Twenty years later, another sterile specimen was collected by $S$. Kamarudin (UKMB) during a medicinal plant survey in 1981. This species is known to occur in Tanah Merah and Jeli, Kelantan, on the Malaysia-Thailand border.

The discovery of new Thottea species sometimes results from entomological studies, due to the use of these plants as food by larvae of swallowtail butterflies (Papilionidae) (Hou 1983). S. Anthonysamy (Universiti Pertanian Malaysia, UPM, now Universiti Putra Malaysia) and J. Weintraub discovered T. anthonysamyi and T. papilionis during the 1987 swallowtail butterfly survey (Kiew pers. comm.). In the same decade, botanical collecting activities carried out by Kiew and Anthonysamy led to the discovery of $T$. praetermissa on the foothills of G. Ledang, Asahan, Malacca and T. ruthiae in Kampung La, Besut, Terengganu.

More recently, T. kamarudiniana was discovered during the Gunung Mandi Angin Scientific Expedition in 2004 (Faridah-Hanum et al. 2006), which was organised by Forestry Department of Peninsular Malaysia (JPSM). This population was in full bloom when found next to a forest track behind a logging camp (Sani pers. comm.). The plants were photographed and some living plants were collected for planting but no flowering material was preserved. Unfortunately, the living material eventually died. On revisiting the collection site, I collected a few fruiting specimens, and made stem cuttings, but they did not survive.

Since the initiation of the Flora of Peninsular Malaysia project in 2005 , field work in botanically less explored areas has led to the discovery of more new species (Kiew \& Rafidah 2007). During several botanical collecting trips to Terengganu, I discovered $T$. piscodora, $T$. reflexa and $T$. terengganuensis. Materials of both $T$. reflexa and $T$. terengganuensis in reproductive stages were first discovered in Lata Tembakah Recreational Forest and Lata Sekayu Recreational Forest, respectively. This shows 
that pristine lowland forests of Peninsular Malaysia still houses species new to science, and recreational forests are playing an important role in biodiversity conservation. Thottea piscodora was found in a disturbed forest adjacent to a freshwater swamp in Rasau Kerteh forest complex.

From examination of the specimens cited by Gamble (1912) deposited in Royal Botanic Garden, Kew (K), I found some drawings on the specimen sheet of Scortechini 1952. The drawings illustrate the ovoid-spherical perianth and annotate the number of stamens and stigmatic lobes of flowers on both Scortechini 1952 and King's Collector 705. These specimens had been identified as $T$. tricornis but the drawings reveal that the specimens actually belong to $T$. anthonysamyi.

Botanists based in the Singapore Botanic Gardens (SING) recently collected a few specimens of Thottea in reproductive stages from the Western Catchment Area, Singapore. These collections prompted me to carefully re-examine the specimen Goodenough 5570 (SING), identified as T. dependens, collected in 1893, and which has a detached inflorescence. The recent collections showed that the inflorescences of this species are positioned at the base of the stem. Flowers preserved in spirit also allowed study of perianth shape and details such as stamens number and arrangement. Now, these specimens can be determined as $T$. praetermissa.

Five out of nine novelties presented here produce inflorescences at the stem base. So, I urge plant collectors when encountering a plant of Thottea to check the stem base for reproductive organs. The shape of perianth and the number and arrangement of stamens are important for species identification. Hence, a good collection must be complemented by flowers preserved in spirit.

\section{NEW TAXA}

1. Thottea anthonysamyi T.L.Yao, sp. nov. - Fig. 1, 10a; Map 1

This species is most similar to $T$. tricornis in the oblanceolate lamina and axillary position of the inflorescence but differs in its flower with 15-20 stamens (not 23-26) and 4-5 stigmatic lobes (not 8-13). It is distinct from all other Thottea species in its ovoid to spherical perianth with perforated apex. - Type: Anthonysamy s.n. (holo SING 15182; iso SING 15178, 15179, 15183), Peninsular Malaysia, Perak, Tapah, disturbed forest in [a] rubber estate, 13 Oct. 1987 (fl and fr).

Thottea tricornis auct. non Maingay ex Hook.f.: Gamble (1912) 29; Ridley (1924) 16; Ding Hou (1984) 74, p.p.

Etymology. Anthonysamy Savarimuthu, born 1943; herbarium assistant and avid plant collector in the Biology Department, University Pertanian Malaysia (now Universiti Putra Malaysia), Serdang, Selangor, Malaysia.

Shrub, 1.5-1.8 m tall, stem slender, erect, scarcely branched, pendent at top. Stems dark green, 4-5 mm diam, surface shallowly furrowed, puberulent; nodes swollen. Leaves: petiole slender, 7-13 mm long, 2.5-3 mm diam, densely puberulent; lamina pale green beneath, drying brown, lanceolate or oblanceolate, $21-25$ by $7.5-10.5 \mathrm{~cm}$, papery to thinly leathery, sparsely pu-

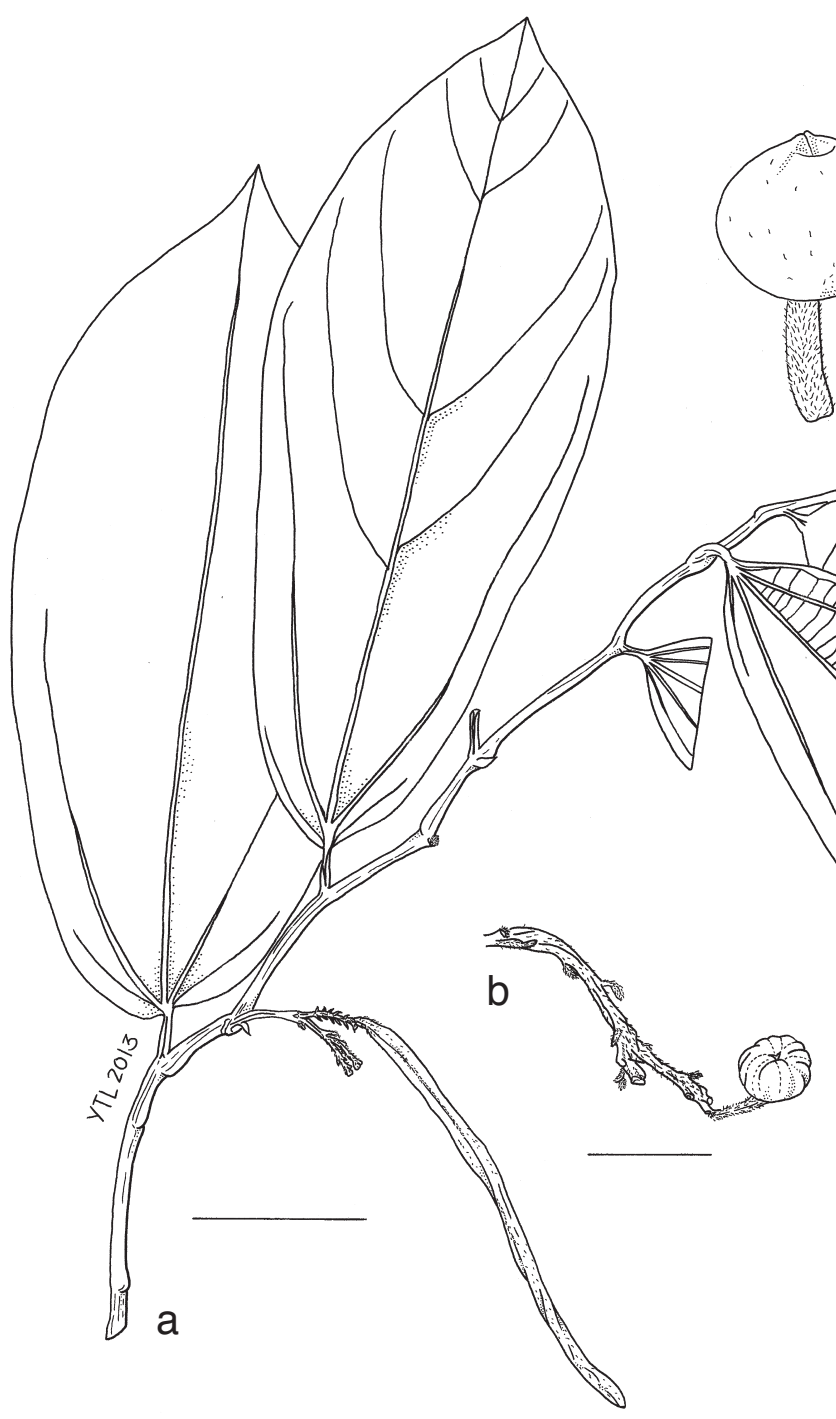
Fig. 1 Thottea anthonysamyi T.L.Yao. a. Habit and a fruiting branch; b. inflo-
rescence; c. flower bud; d. flower bud with perianth partly removed showing gynostemium. - Scale bars: $a=5 \mathrm{~cm} ; b=1 \mathrm{~cm} ; \mathrm{c}, \mathrm{d}=2 \mathrm{~mm}$ (a: Anthonysamy s.n., SING 15178; b: Anthonysamy s.n., SING 15182; c, d: Anthonysamy s.n., SING 15179). 


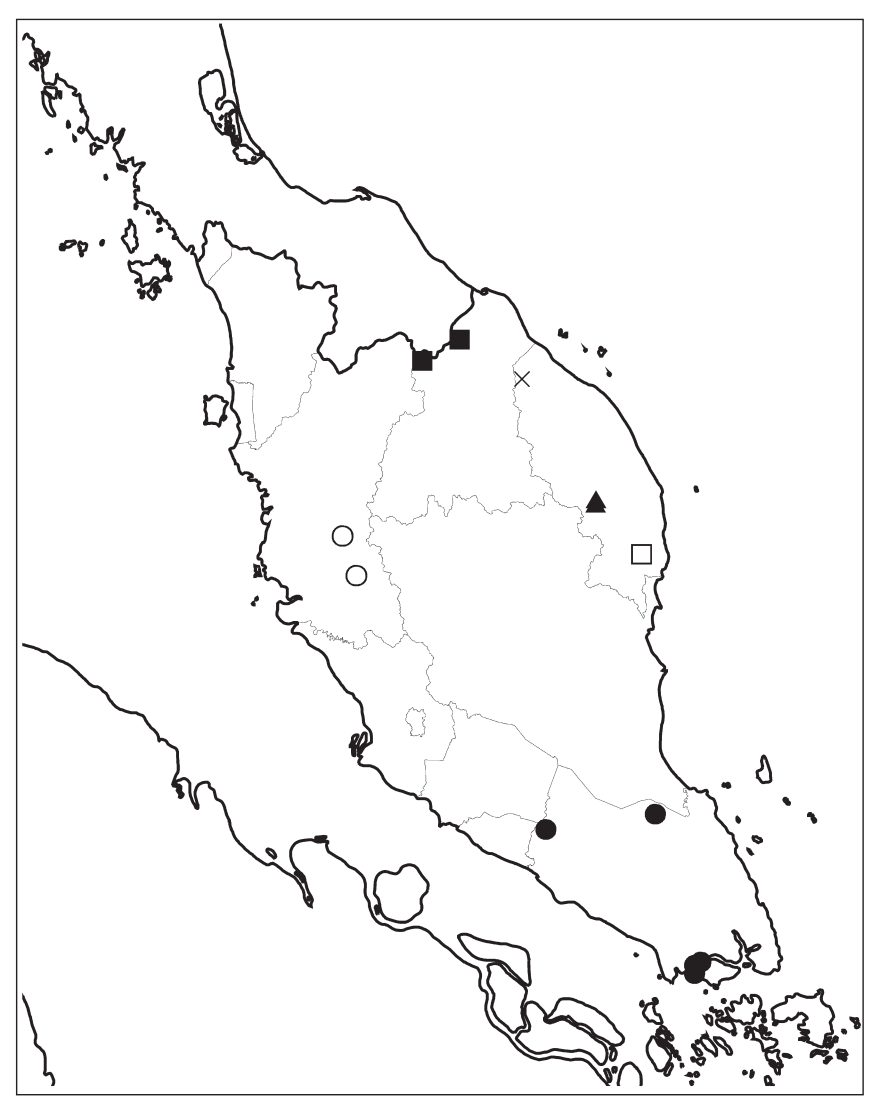

Map 1 Distribution of Thottea anthonysamyi (O), T. kamarudiniana ( $\mathbf{\Delta}), T$. longipedunculata $(\square)$, T. piscodora $(\square)$, T. praetermissa $(\bullet)$ and T. reflexa $(\times)$.

berulent beneath, base cuneate, margin entire, apex blunt or acute; midrib above pronounced, below prominent, venation pinnate with basal pairs, above noticeable, below prominent, basal pairs 2, upper basal pair less than, or sometimes more than half of the lamina length, lateral pairs 5-6; intercostal veins a mix of ladder-like and net-like, ladder-like veinlets more pronounced. Inflorescences in axils of foliage leaf, congregated or solitary; peduncle sometimes once branched, 13-24 mm long, c. $2 \mathrm{~mm}$ diam, pubescent; bracts lanceolate, c. 3 by 1.5 $\mathrm{mm}$, densely pubescent, apex blunt, venation obscure. Flowers erect; pedicel and ovary c. $0.6 \mathrm{~cm}$ long, ovary c. $1 \mathrm{~mm}$ diam, densely pubescent; perianth creamy white, ovoid to spherical, obscurely 3-lobed, top axis perforate, c. $0.4 \mathrm{~cm}$ long, c. $0.7 \mathrm{~cm}$ diam, venation faint, outer surface puberulent, glabrescent, inner surface puberulent; stamens 15-20 in 2 whorls, upper whorl with 5-6 stamens, lower whorl 10-14, filamentous; filaments c. $0.5 \mathrm{~mm}$ long, glabrous, anthers positioned at the swollen connective apex, oblong, c. $0.5 \mathrm{~mm}$ long, c. $0.2 \mathrm{~mm}$ wide; style column cylindric, c. $1 \mathrm{~mm}$ long, glabrous; stigmatic lobes 4-5, positioned higher than stamens, c. $1 \mathrm{~mm}$ long, glabrous. Capsules pendent, ripening brown, twisted, 4-angled, 125-145 $\mathrm{mm}$ long, 3-6 mm diam, densely puberulent. Seeds ellipsoid, c. 4 by $2 \mathrm{~mm}$, 3-angled, slightly ridged, margin corrugated, testa surface wrinkled, apex and base acute.

Distribution - Endemic to Peninsular Malaysia, known only from Perak.

Ecology - Lowland forest.

Conservation status - Endangered B2ab(iii). This species has not been collected in the past 20 years. Both known localities have been severely disturbed.

Additional specimens examined. Peninsular Malaysia, Perak, Gopeng [Goping], King's Collector 705, Sept. 1880 (K); Scortechini 1952, Apr. 1885 (K).

Notes -1 . The white spherical-ovoid perianth of this species is unique in Thottea. The only opening is an aperture at its top axis, which presumably is the entrance for insect pollinators. The largest flower available for this study might not be a fully developed one.

2. Vegetative characters and the compact inflorescence when young resemble those of $T$. tricornis.

3. For comparison of $T$. anthonysamyi and T. tricornis see Table 1.

\section{Thottea kamarudiniana T.L.Yao, sp. nov. - Fig. 2, 10b; Map 1}

This species is similar to $T$. grandiflora in its broadly lanceolate lamina and the inflorescence that is positioned at the stem nodes below foliage leaves but well above ground level but it is different in its perianth that has three deeply dissected lobes with a shallow cupular base (not bell-shaped perianth). - Type: Sani et al. SM 449 (holo UKMB, with a colour photograph of flowers), Peninsular Malaysia, Terengganu, Pasir Raja FR [Kampung Pasir Raja], track to big Chengal [tree], 6 June 2004 (fl).

Etymology. Kamarudin Mat Salleh, affectionately known as Pak Din, 1959-2009; a Malaysian botanist and Professor in Universiti Kebangsaan Malaysia, best known for his research on Rafflesia (Rafflesiaceae). He passed away prematurely.

Shrub, 1.3-2.5 m tall, stem stout, erect, scarcely branched, pendent at top. Stems dark green, 8-10 mm diam, surface shallowly furrowed, densely pubescent; nodes indistinct. Leaves: bract-like reduced leaves 11-19, foliage leaves c. 7 on one stem; petiole stout, 12-14 $\mathrm{mm}$ long, 4-5 mm diam, densely pubescent; lamina dark green above, green beneath, drying brown, broadly lanceolate, $43-44$ by $26-27 \mathrm{~cm}$, first foliage lamina distinctly smaller, papery to thinly leathery, indumentum beneath densely pubescent, base rounded or cordate, margin entire, apex acute; midrib above flat, below prominent; venation pinnate with basal pairs, above noticeable, below prominent, basal pairs 2, upper basal pair less than half of the leaf length, lateral pairs 8-12; intercostal veins a mix of ladder-like and net-like, ladder-like veinlets more pronounced. Inflorescences on stem below foliage leaves well above ground level, solitary; peduncle sometimes once branched, c. $45 \mathrm{~mm}$ long, c. $4 \mathrm{~mm}$ diam, densely pubescent; bracts light green, lanceolate, 10-12 by $4-6 \mathrm{~mm}$, pubescent, apex acute, venation distinct. Flowers pendent; perianth creamy white on outer surface, venation dis-

Table 1 Comparison of Thottea anthonysamyi and T. tricornis.

\begin{tabular}{lll}
\hline & T. anthonysamyi & T. tricornis \\
\hline Lamina: & lanceolate or oblanceolate: & broadly ovate, narrowly oblanceolate or oblanceolate: \\
length by width $(\mathrm{cm})$ & $21-25$ by $7.5-10.5$ & $3-6.5-22-36.5$ by $8-16.5$ \\
Peduncle length $(\mathrm{cm})$ & $1.3-2.4$ & magenta on outer surface, creamy white on inner surface \\
Perianth colour & creamy white & 3-lobed, funnel-shaped, base not distinctly contracted; lobes \\
Perianth shape & ovoid to spherical, top axis perforate and obscurely & faintly deltoid, flared \\
Perianth length by diam $(\mathrm{cm})$ & 0.4 by 0.7 (not fully mature) & $1.4-1.5$ by 1.5-3.5 \\
Stamens & $15-20$ in 2 whorls; upper whorl 5-6, lower whorl 10-14 & $23-26$ in 2 whorls, upper whorl 9-10, lower whorl 14-16 \\
Stigmatic lobe number & $4-5$ & $8-13$ \\
\hline
\end{tabular}


Table 2 Comparison of Thottea kamarudiniana and T. grandiflora.

\begin{tabular}{|c|c|c|}
\hline & T. kamarudiniana & T. grandiflora \\
\hline Lateral vein pairs & $8-12$ & $(5-) 6-8(-10)$ \\
\hline Inflorescences & $\begin{array}{l}\text { at nodes of stem below foliage leaves well above } \\
\text { ground level }\end{array}$ & $\begin{array}{l}\text { at axils of foliage leaf or at nodes of stem below foliage } \\
\text { leaves well above ground level }\end{array}$ \\
\hline Perianth colour & $\begin{array}{l}\text { creamy white on outer surface, veins claret, creamy } \\
\text { white on inner surface }\end{array}$ & dark claret, veins white on outer surface \\
\hline Perianth shape & $\begin{array}{l}\text { 3-lobed, lobes deeply dissected almost to the base, base } \\
\text { contracted into a shallow cup; lobes broadly ovate, flare }\end{array}$ & bell-shape, 3-lobed; lobes oblong, rarely deltoid \\
\hline
\end{tabular}

tinct, claret-coloured, creamy white on inner surface, 3-lobed, lobes deeply dissected almost to the base, base contracted into a shallow cup; perianth lobes broadly ovate, flared, apex acute. Capsules pendent, ripening brown, twisted, 4-angled, c. $13 \mathrm{~cm}$ long, ferruginous villose. Seed unknown.

Distribution - Endemic to Peninsular Malaysia, known only from Pasir Raja, Terengganu.
Ecology — Lowland forest in a tree-fall gap at $160 \mathrm{~m}$ altitude, and from edge of trail behind logging camp and beside a wild boar wallow.

Conservation status - Critically Endangered B2ab(iii). This species is only known from its type locality and no population occurs within the network of Totally Protected Area.

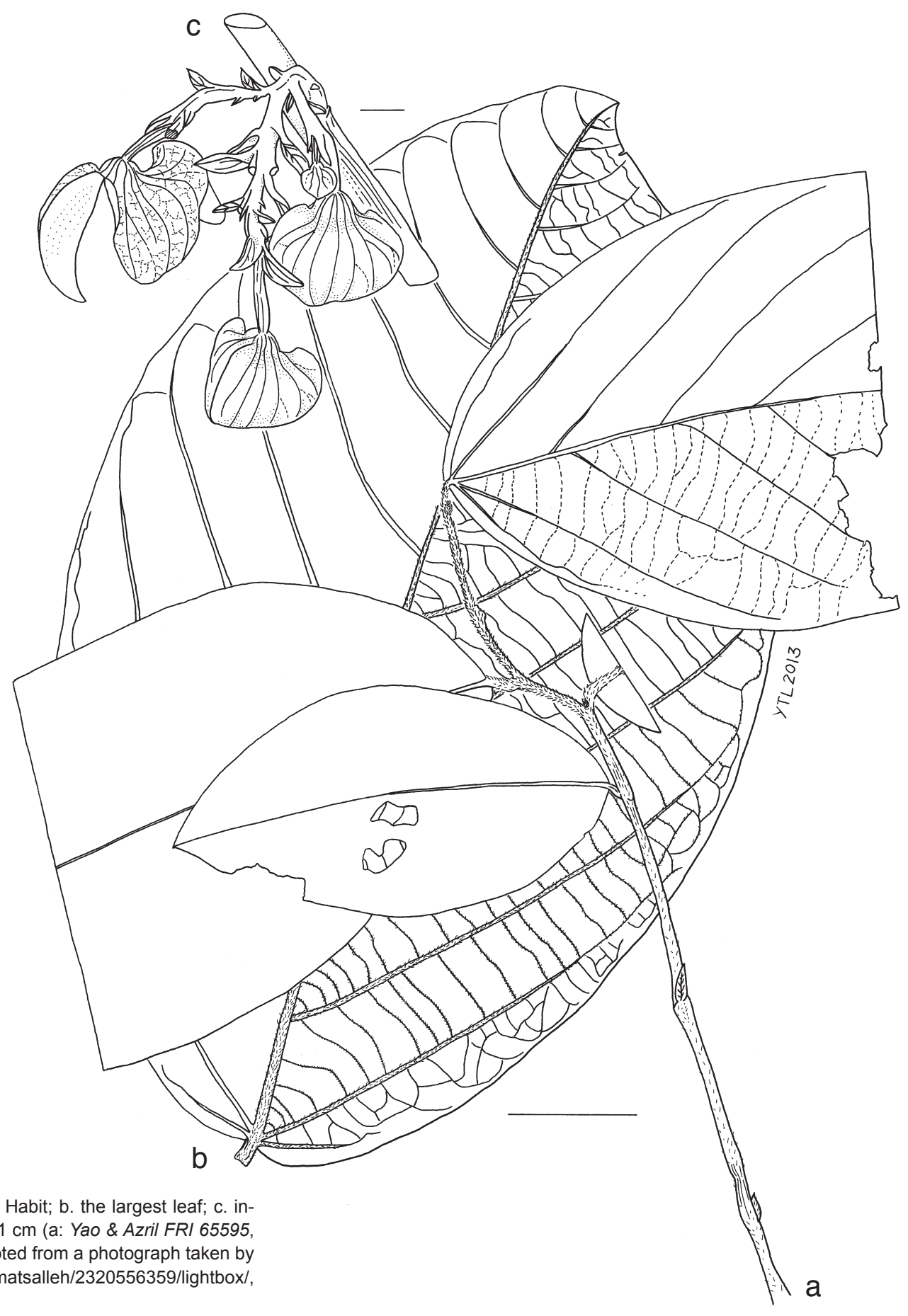

Fig. 2 Thottea kamarudiniana T.L.Yao. a. Habit; b. the largest leaf; c. inflorescence. - Scale bars: $a, b=\mathrm{cm} ; \mathrm{c}=1 \mathrm{~cm}$ (a: Yao \& Azril FRI 65595, KEP; b: Sani et al. SM 449, UKMB; c: adapted from a photograph taken by S. Kamarudin (http://m.flickr.com/photos/kmatsalleh/2320556359/lightbox/, last accessed 1 Aug. 2013)). 
Table 3 Comparison of Thottea longipedunculata and T. sumatrana.

\begin{tabular}{lll}
\hline & T. longipedunculata & T. sumatrana \\
\hline Lamina: & narrowly ovate or oblong: & narrowly ovate or lanceolate: \\
length by width $(\mathrm{cm})$ & $24.5-30$ by $9.5-10$ & $20-21.5$ by $7-9.5$ \\
Lateral vein pairs & $7-8$ & $4-6(-8)$ \\
Peduncle length $(\mathrm{cm})$ & c. 28 & $7.5-10.5$ \\
Bracts arrangement & lax & crowded \\
Perianth colour & white and red & cream on outer surface, dull crimson on inner surface \\
Perianth shape & 3-lobed; lobes shallowly deltoid & 3-lobed, lobes deeply dissected to the base, base not distinctly \\
Perianth length by diam $(\mathrm{cm})$ & c. 1.5 by 1.6 & contracted; lobes oblong, flare \\
Stamens & 20 in 2 whorls; upper whorl 8, lower whorl 12 & c. 3.5 by 3.5 \\
Stigmatic lobe number & c. 6 & consistently 6 in 1 whorl \\
\hline
\end{tabular}

Additional specimen examined. Peninsular Malaysia, Terengganu, Pasir Raja FR, Compartment 5, Yao \& Azril FRI 65995 (K, KEP 192700, 192701, 192702, L), 28 June 2011 (fr).

Notes - 1 . The type is a two sheets accession with a colour photograph of inflorescence with flowers in full bloom, collected during Gunung Mandi Angin Expedition organised by Forestry Department of Peninsular Malaysia. The shape of perianth is described from the image but details such as stamen count are not known. The perianth shape of this species resembles T. straatmanii Ding Hou in Sumatra. The distribution of these two species is disjunct though.

2. For comparison of T. kamarudiniana and T. grandiflora see Table 2.

3. Thottea longipedunculata T.L.Yao, sp. nov. - Fig. 3, 10c; Map 1

This species is similar to $T$. sumatrana in its narrowly ovate lamina with a rounded base but is different in the exceptionally long peduncle c. $28 \mathrm{~cm}$ (not to $10.5 \mathrm{~cm}$ ), the perianth with shallowly deltoid lobes (not oblong ones) and
20 stamens in two whorls (not consistently 6 in one whorl). - Type: Motan KEP 94538 (holo KEP 78121), Peninsular Malaysia, Kelantan, Tanah Merah, Kemahang FR, Compartment 65, 13 July 1960 (fl).

Etymology . Latin, longus = long, pedunculus = peduncle; referring to the exceptionally long inflorescence.

Shrub, c. $1.8 \mathrm{~m}$ tall, stem slender, erect, scarcely branched, pendent at top. Stems dark green, c. $7.5 \mathrm{~mm}$ diam, surface smooth, pubescent; nodes indistinct, above nodes constricted. Leaves: petiole rather stout, 8-10 $\mathrm{mm}$ long, 2-4 $\mathrm{mm}$ diam, pubescent; lamina drying brown, narrowly ovate or oblong, 24.5-30 by 9.5-10 cm, papery, indumentum beneath pubescent, base cuneate or rounded, margin entire, apex acute or shortly mucronate; midrib above flat or sometimes sunken, prominent beneath; venation pinnate with basal pairs, above slightly sunken, below prominent, basal pairs 2, upper basal pair less than half of the leaf length, lateral pairs 7-8; intercostal veins a mix of ladder-like and net-like, ladder-like veinlets more pronounced. Inflorescence position unknown, solitary; peduncle once branched, c. $280 \mathrm{~mm}$ long, c. $3 \mathrm{~mm}$ diam, pubescent; bracts
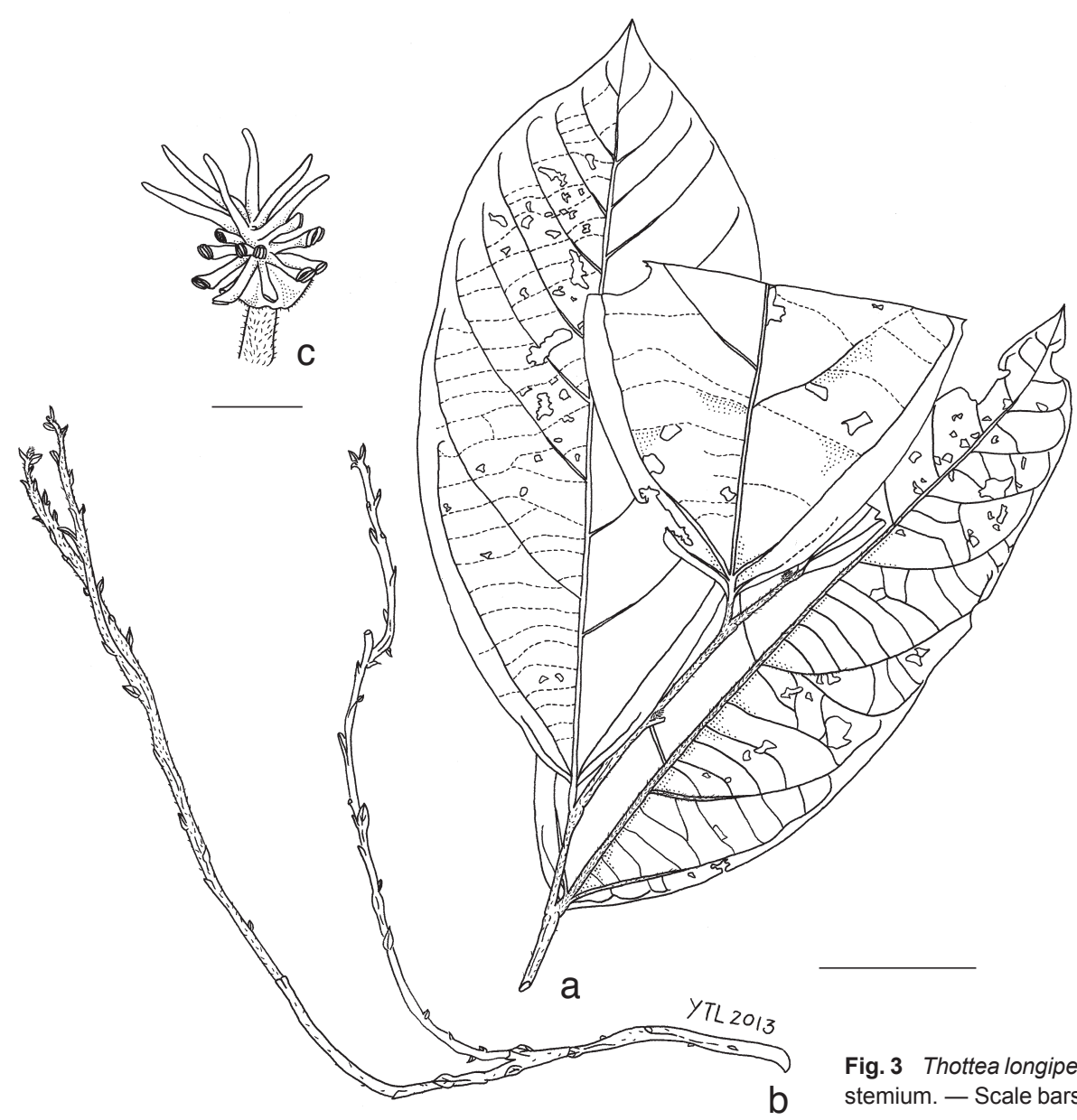

Fig. 3 Thottea longipedunculata T.L.Yao. a. Habit; b. inflorescence; c. gynostemium. - Scale bars: $a, b=5 \mathrm{~cm} ; \mathrm{c}=2 \mathrm{~mm}$ (all: Motan KEP 94538, KEP). 
ovate, c. 11 by $5 \mathrm{~mm}$, pubescent, apex blunt or acute, venation obscure or sometimes distinct. Flowers: ovary densely pubescent; perianth white and red, 3-lobed, c. $1.5 \mathrm{~cm}$ long, c. 1.6 $\mathrm{cm}$ diam, outer surface pubescent, inner surface sparsely pubescent; perianth lobes shallowly deltoid, c. 5 by $10 \mathrm{~mm}$, apex blunt; stamens c. 20 in 2 whorls, upper whorl with c. 8 stamens, lower whorl c. 12, filamentous; filament c. $1 \mathrm{~mm}$ long, glabrous; anthers positioned at the swollen connective apex, oblong, c. 0.5 $\mathrm{mm}$ long, c. $0.2 \mathrm{~mm}$ wide; style column cylindric, c. $1.5 \mathrm{~mm}$ long, glabrous; stigmatic lobes c. 6 , positioned higher than stamens, c. $2.5 \mathrm{~mm}$ long, glabrous. Capsule and seed unknown.

Vernacular name - Hempedu beruang (Malay).

Distribution - Endemic to Peninsular Malaysia. It is known only from Tanah Merah, Kelantan.

Ecology - Forest undergrowth.

Conservation status - Critically Endangered B1ab(iii). This species has not been collected in the past 30 years. Both known localities have been severely disturbed.

Additional specimen examined. Peninsular Malaysia, Kelantan, Tanah Merah, Batu Melintang, Belimbing, Batu Tekok, Kamarudin KMS 139 (UKMB 11516), 4 July 1981.

Note - For comparison of T. longipedunculata and T. sumatrana see Table 3.

\section{Thottea papilionis T.L.Yao, sp. nov. - Fig. 4, 10d; Map 2}

This species is similar to $T$. rhizantha in the inflorescence positioned at the stem base, in its claret-violet and white-cream perianth, 3-lobed perianth with a constricted base and 20-24 stamens arranged in two whorls, but this species differs in its smaller leaves $15.5-17.5$ by $4-5 \mathrm{~cm}$ (not 21-42.5 by $9.5-16.5 \mathrm{~cm}$ ) and shorter bowl-shaped perianth base $5-7 \mathrm{~mm}$ long (not c. $15 \mathrm{~mm}$ ). This species is also similar to $T$. dependens in its oblanceolate lamina with a mucronate apex, 3-lobed perianth with bowl-shape base and stamens arranged in two whorls but it is different in its green lamina under surface (not glaucous), distinct intercostal veins (not faint), inflorescence position at base of stem close to ground level (not at stem axils well above ground level) and claret perianth with a creamy white patch on each perianth lobe (not plain magenta). - Type: Yao et al. FRI 65421 (holo KEP 192442; iso K, L), Peninsular Malaysia, Perak, Tapah, orchard on Sungai Batang Padang north bank, 31 Mar. 2009 (fl).

Etymology. Latin, papilio = butterfly; of butterfly, it is a food plant for butterfly pupae.

Shrub, stem slender, erect, scarcely branched, pendent at top; c. $1 \mathrm{~m}$ tall. Stems dark green, c. $6.5 \mathrm{~mm}$ diam, surface smooth, puberulent; nodes pronounced, above nodes constricted. Leaves: foliage leaves $12-19$ on one stem; petiole slender, 7-8 mm long, 1.5-2 mm diam, densely puberulent; lamina green above, pale green beneath, drying brown, oblanceolate, $16-18$ by $3.5-5 \mathrm{~cm}$, first foliage lamina distinctly smaller, nar-

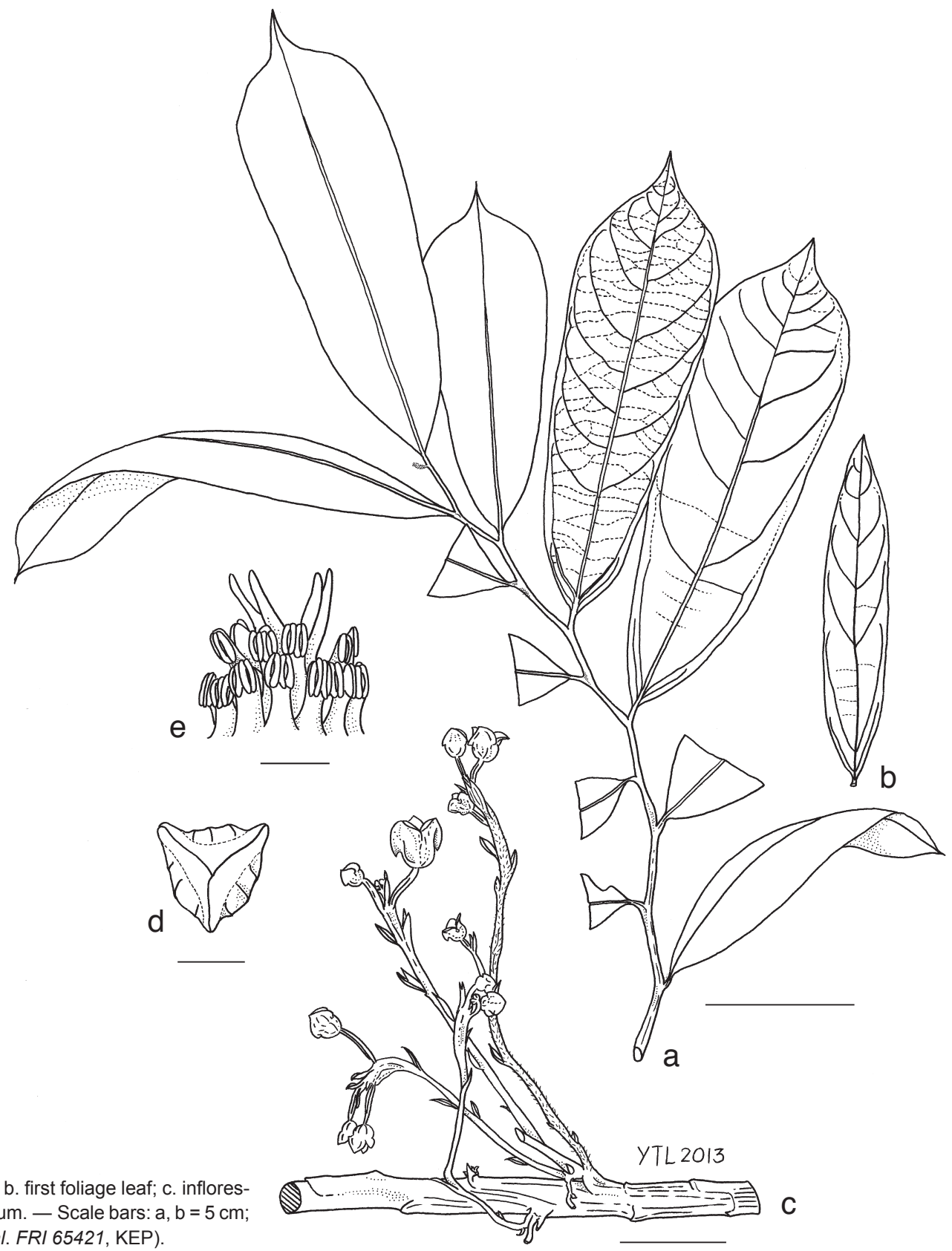

Fig. 4 Thottea papilionis T.L.Yao. a. Habit cence; d. flower bud, top view; e. gynostemium. - Scale bars: $a, b=5 \mathrm{~cm}$ $\mathrm{c}=2 \mathrm{~cm} ; \mathrm{d}=5 \mathrm{~mm} ; \mathrm{e}=2 \mathrm{~mm}$ (all: Yao et al. FRI 65421, KEP). 
Table 4 Comparison of Thottea papilionis, T. dependens and T. rhizantha.

\begin{tabular}{|c|c|c|c|}
\hline & T. papilionis & T. dependens & T. rhizantha* \\
\hline $\begin{array}{l}\text { Lamina: } \\
\text { length by width }(\mathrm{cm})\end{array}$ & $\begin{array}{l}\text { oblanceolate: } \\
16-18 \text { by } 3.5-5\end{array}$ & $\begin{array}{l}\text { ovate, obovate, oblong or oblanceolate: } \\
(17-) 19.5-28.5(-31) \text { by }(5.5-) 8.5-13(-16)\end{array}$ & $\begin{array}{l}\text { elliptic, oblanceolate or oblong: } \\
21-42.5 \text { by } 9.5-16.5\end{array}$ \\
\hline Lamina base & cuneate & cuneate or rounded & cuneate or obtuse \\
\hline Lamina apex & mucronate, sometimes acute & acute or acuminate, sometimes mucronate & acuminate \\
\hline Inflorescences & at base of stem & $\begin{array}{l}\text { at nodes of stem below foliage leaves well } \\
\text { above ground level }\end{array}$ & at base of stem \\
\hline Peduncle length $(\mathrm{cm})$ & $7.5-9$ & $2.8-4.5(-6)$ & c. 13 \\
\hline Perianth colour & $\begin{array}{l}\text { claret with a creamy white patch on } \\
\text { each perianth lobe }\end{array}$ & dark magenta & $\begin{array}{l}\text { outer surface faintly violet tinged, inner surface } \\
\text { violet at the base, white at the top or red and white }\end{array}$ \\
\hline Perianth shape & $\begin{array}{l}\text { 3-lobed, base contracted into a bowl- } \\
\text { shaped cup } 5-7 \mathrm{~mm} \text { long; lobes } \\
\text { broadly ovate, flared }\end{array}$ & $\begin{array}{l}\text { 3-lobed, base contracted into a bowl- } \\
\text { shaped cup; lobes broadly ovate, flared }\end{array}$ & $\begin{array}{l}\text { funnel-shape, base cylindric tubular, c. } 15 \mathrm{~mm} \\
\text { long; lobes broadly ovate, spreading }\end{array}$ \\
\hline $\begin{array}{l}\text { Perianth length by } \\
\text { diam }(\mathrm{cm})\end{array}$ & $1.3-1.6$ by $1.3-2$ & $(0.8-) 1.5-2$ by $(1-) 2$ & $3-3.5$ by 3.5 \\
\hline Stamens & $\begin{array}{l}20-24 \text { in } 2 \text { whorls; upper whorl } 7-9 \text {, } \\
\text { lower whorl } 13-15\end{array}$ & $\begin{array}{l}(21-) 28-30 \text { in } 2 \text { whorls; upper whorl } \\
(8-) 11-12, \text { lower whorl }(13-) 17-18\end{array}$ & $\begin{array}{l}19-23 \text { in } 2 \text { whorls; upper whorl } 6-8 \text {, } \\
\text { lower whorl } 13-15\end{array}$ \\
\hline
\end{tabular}

* Attributes of the characters were based on species description and illustrations of Plantae Beccarianae 3793 (Beccari 1870), and species description in Flora Malesiana (Hou 1984).

rowly lanceolate, c. 11.3 by $2 \mathrm{~cm}$, papery, indumentum beneath sparsely puberulent, base cuneate, margin entire, apex acute or mucronate; midrib above flat, below prominent; venation pinnate with basal pairs, above faint, below noticeable, basal pairs 2 , upper basal pair less than half of the leaf length, lateral pairs $5-7$; intercostal veins a mix of ladder-like and net-like, ladderlike veinlets more pronounced. Inflorescences at stem base close to ground level, congregated; peduncle not branched, 75-90 mm long, c. $1 \mathrm{~mm}$ diam, densely puberulent; bracts narrowly ovate, $6-7$ by $2-3 \mathrm{~mm}$, pubescent, apex acute, venation distinct. Flowers erect; pedicel and ovary 1-1.2 cm long, ovary c. $1 \mathrm{~mm}$ diam, densely pubescent; perianth claret-coloured with a creamy white patch on each perianth lobe, 3-lobed, 1.3-1.6 $\mathrm{cm}$ long, 1.3-2 cm diam, venation distinct, outer surface sparsely pubescent, inner surface puberulent; base contracted

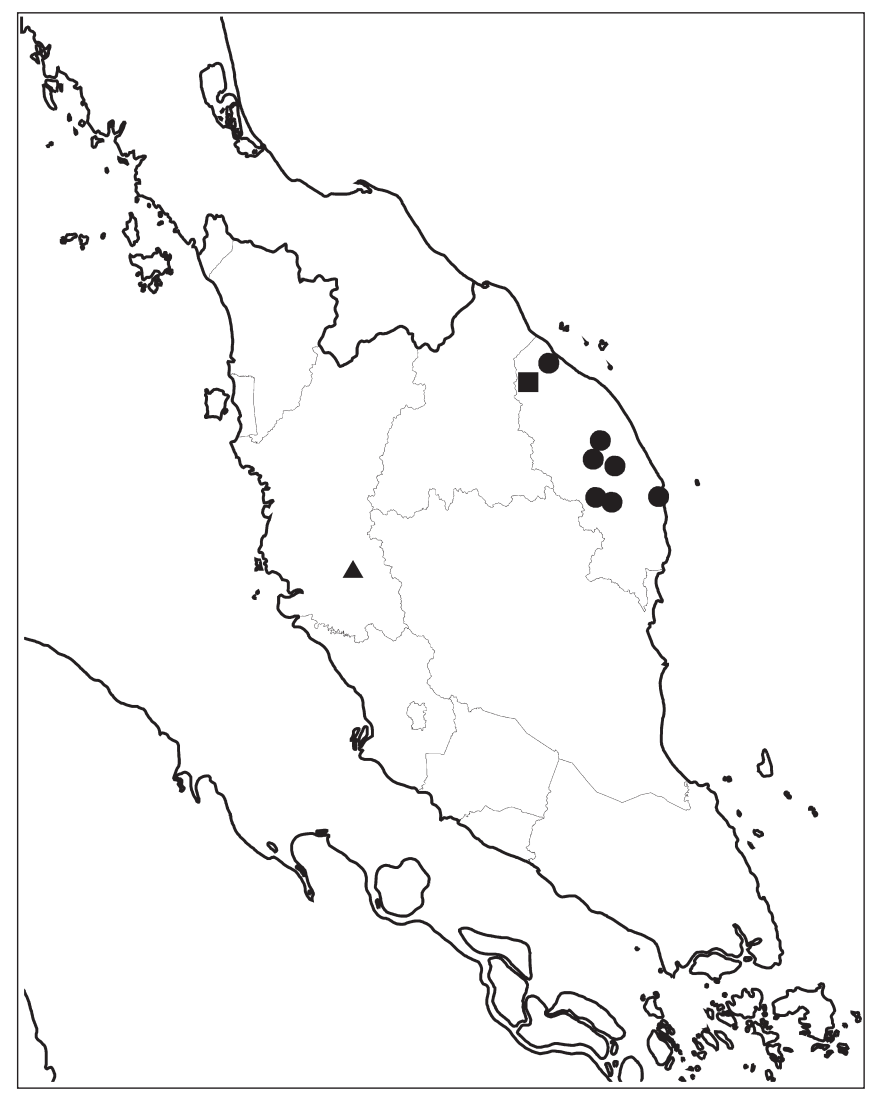

Map 2 Distribution of Thottea papilionis ( $\mathbf{\Delta})$, T. ruthiae $(\boldsymbol{\square})$ and T. terengganuensis (O). into a bowl-shaped cup, $0.5-0.7 \mathrm{~cm}$ deep, c. $0.7 \mathrm{~cm}$ diam, inner surface pubescent; perianth lobes broadly ovate, flared, 8-10 by c. $13 \mathrm{~mm}$, apex blunt or acute; stigmatic lobes positioned higher than stamens; stamens 20-24 in 2 whorls, upper whorl with 7-9 stamens, lower whorl 13-15, filamentous; filament c. $1 \mathrm{~mm}$ long, glabrous; anthers positioned at swollen connective apex, oblong, c. $1.2 \mathrm{~mm}$ long, c. $0.6 \mathrm{~mm}$ wide; style column disc-shaped, c. $2.6 \mathrm{~mm}$ long, glabrous; stigmatic lobes 4-6, c. $1.8 \mathrm{~mm}$ long, glabrous. Capsule and seed unknown.

Distribution - Endemic to Peninsular Malaysia, known only from Tapah, Perak.

Ecology - Lowland forest. Planted, natural habitat unknown, collected from a mix fruit orchard.

Conservation status - Critically Endangered B2ab(iii). This species is only known from the type specimen and no population occurs within the network of Totally Protected Area.

Notes - 1. Known only from the type and a photograph, which was published (Polunin 2004) as an unnamed Thottea sp.

2. For comparison of T. papilionis, T. dependens and T. rhizantha see Table 4.

\section{Thottea piscodora T.L.Yao, sp. nov. - Fig. 5, 10e; Map 1}

This species is similar to $T$. dependens in its glaucous lamina under surface, faint intercostal veins, inflorescence produced below foliage leaves but well above ground level and stamens arrange in two whorls but it is different in its urn-shape perianth base (not bowl-shape), fewer stamens 14-15 (not 21-30) and creamy white perianth (not plain magenta). - Type: Yao et al. FRI 65443 (holo KEP 192446; iso K, L, SING), Peninsular Malaysia, Terengganu, Kemaman, Rasau Kerteh FR, 8 Apr. 2009 (fl).

Etymology. Latin, piscis = fish, odor = smell; denoting the flower with a rotten fish smell.

Shrub, c. 2 m tall, stem stout, erect, scarcely branched, pendent at top. Stems dark green, 4-6 $\mathrm{mm}$ diam; nodes pronounced. Leaves: petiole rather stout, c. $7 \mathrm{~mm}$ long, c. $4.5 \mathrm{~mm}$ diam; lamina dark green above, distinctly glaucous beneath, narrowly lanceolate, $25-28.5$ by $7.5-9.5 \mathrm{~cm}$, first foliage lamina distinctly smaller, narrowly ovate, $11-15.5$ by $1-2.5 \mathrm{~cm}$, thinly leathery, glabrous beneath, base cuneate, margin entire, apex acute; midrib above flat or slightly raised, below prominent; venation pinnate with basal pairs, noticeable above and below, basal pairs 2, upper basal pair less than half of the leaf length, lateral pairs 5-8; intercostal veins a mix of ladder-like and net-like, ladder-like veinlets more pronounced, net-like veinlets obscure. Inflorescences on stem below foliage leaves well above ground level, congregated, a few inflorescences produced from node thicken due to repeating flowering; peduncle sometimes once 
branched, 0.4-0.8 mm long, c. $1.6 \mathrm{~mm}$ diam, glabrescent; bracts oblanceolate or ovate, c. 2 by $1 \mathrm{~mm}$, glabrescent, apex acute or sometimes notched or bifid, venation obscure. Flowers: ovary ascending, perianth horizontally held; pedicel and ovary c. $2 \mathrm{~cm}$ long, ovary c. $1.5 \mathrm{~mm}$ diam, pubescent; perianth creamy white, towards centre tinged pale greenish grey, 3-lobed, c. $1.2 \mathrm{~cm}$ long, c. $0.9 \mathrm{~cm}$ diam, venation distinct, outer surface puberulent, inner surface sparsely puberulent; base contracted into an urn-shaped cup, c. $0.5 \mathrm{~cm}$ deep, c. $0.7 \mathrm{~cm}$ diam, inner surface pubescent; perianth lobes broadly ovate, flared, c. 7 by $5 \mathrm{~mm}$, apex acute; stamens $14-15$ in 2 whorls, upper whorl with 5-6 stamens, lower whorl c. 9, filamentous; filament c. $1 \mathrm{~mm}$ long, puberulent; anthers positioned at the swollen connective apex, oblong, c. $1.2 \mathrm{~mm}$ long, c. $0.4 \mathrm{~mm}$ wide; style column cylindric, $2 \mathrm{~mm}$ long, glabrous; stigmatic lobes $4-5$, positioned higher than stamens, c. $1.5 \mathrm{~mm}$ long, glabrous. Capsule and seed unknown.

Distribution - Endemic to Peninsular Malaysia, known only from Rasau Kerteh, Terengganu.

Ecology - Lowland forest, forest fringe close to swamp. Flies are attracted to the flowers that smell of rotten fish.

Conservation status - Critically Endangered B2ab(iii). The forest of the single known locality has been severely disturbed.

\section{Thottea praetermissa T.L.Yao, sp. nov. - Fig. 6, 10f; Map 1}

This species is closely allied to $T$. beccarii in inflorescence position at stem base, continuum of stamen number range from 24-30 ( $T$. beccarii 20-24) arranged in two whorls, continuum of stigmatic lobe number from 11-13 (c. 10) but this species differs in its inflorescence with laxly arranged bracts (not crowded) and saucer-shape perianth (not cupular). This species is also similar to $T$. dependens in its oblong lamina with cuneate base, acute and sometimes shortly mucronate apex and 24-30 stamens arranged in two whorls but it is different in the green lamina lower surface (never glaucous), distinct intercostal veins (not faint), inflorescences positioned at stem base close to, or with a few well above ground level (not always well above ground level) and the saucer-shaped perianth (not with bowl-shaped cupular base). - Type: Anthonysamy SA 917 (holo KEP 192448; iso SING 15180), Peninsular Malaysia, Malacca, Asahan, 24 Aug. 1987 (fl and fr).

Thottea dependens auct. non (Planch.) Klotzsch: Ridley (1900) 127; Gamble (1912) 28; Ridley (1924) 16; Ding Hou (1984) 75, p.p.

Thottea tricornis auct. non Maingay ex Hook.f.: Ding Hou (1984) 74, p.p.

Etymology. Latin, praetermissus = overlooked; referring to this overlooked species. First collected in 1893 but only recognised here as a distinct species.

Shrub, 0.5-1.3 m tall, stem slender, erect, scarcely branched, pendent at top. Stems dark green, 4-5 mm diam, surface smooth, densely puberulent; nodes pronounced. Leaves: bract-like reduced leaves $4-5$, foliage leaves (4-)7-9 on one stem; petiole

Note - For comparison of T. piscodora and T. dependens see Table 5.

Fig. 5 Thottea piscodora T.L.Yao. a. Habit; b. first foliage leaf; c. inflorescences; $d$. inflorescence; e. gynostemium. - Scale bars: $a, b=5 \mathrm{~cm} ; c=4$ $\mathrm{cm}, \mathrm{d}=1 \mathrm{~cm} ; \mathrm{e}=2 \mathrm{~mm}$ (all: Yao et al. FRI 65443, KEP).

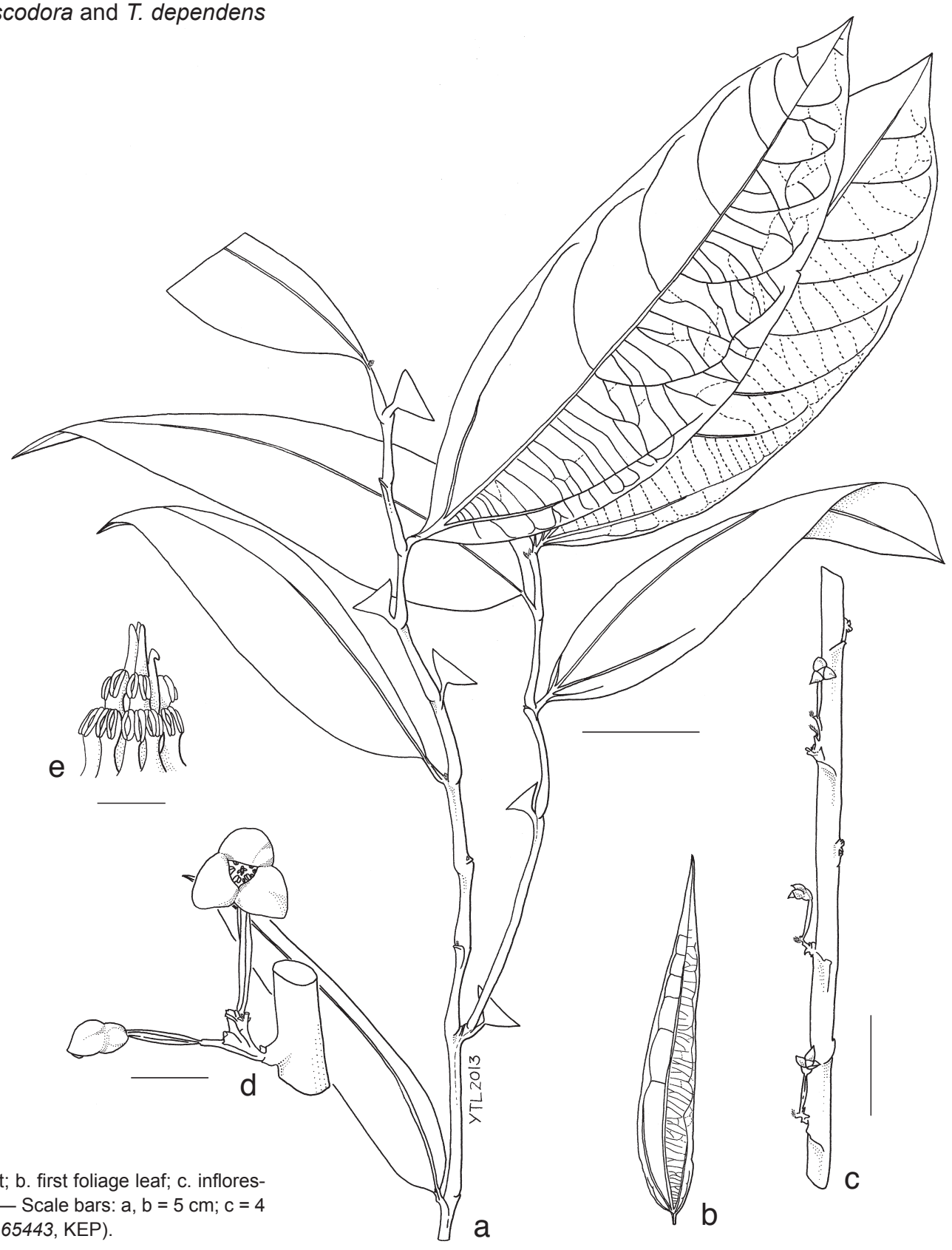


Table 5 Comparison of Thottea piscodora and T. dependens.

\begin{tabular}{lll}
\hline & T. piscodora & T. dependens \\
\hline Lamina: & narrowly lanceolate: & ovate, obovate, oblong or oblanceolate: \\
length by width $(\mathrm{cm})$ & $25-28.5$ by $7.5-9.5$ & $(17-) 19.5-28.5(-31)$ by $(5.5-) 8.5-13(-16)$ \\
Lamina lower surface colour & distinctly glaucous & pale green, sometimes glaucous \\
Perianth colour & creamy white, towards central tinged pale greenish grey & dark magenta \\
Perianth shape & 3-lobed, base contracted into an urn-shaped cup; & 3-lobed, base contracted into a bowl-shaped cup; \\
& lobes broadly ovate, flare & lobes broadly ovate, flare \\
Perianth length by diam $(\mathrm{cm})$ & 1.2 by 0.9 & $(0.8-) 1.5-2$ by (1-)2 \\
Stamens & $14-15$ in 2 whorls; upper whorl 5-6, lower whorl 9 & $(21-) 28-30$ in 2 whorls; upper whorl (8-)11-12, lower whorl (13-)17-18 \\
\hline
\end{tabular}

slender, 5-10 mm long, 1-3 mm diam, puberulent; lamina green above, pale green beneath, drying brown, oblong or lanceolate, $15-22$ by $5-7.5 \mathrm{~cm}$, first foliage lamina sometimes distinctly smaller, narrowly lanceolate, c. 11 by $2 \mathrm{~cm}$; thinly leathery, indumentum beneath sparsely puberulent, base cuneate, margin entire, apex acute or shortly mucronate; midrib above flat or sometimes sunken, below prominent; venation pinnate with basal pairs, noticeable above and below, basal pairs 2 , upper basal pair less than, or sometimes more than half of the lamina length, lateral pairs 5-6; intercostal veins a mix of ladder-like and net-like, ladder-like veinlets more pronounced. Inflorescences at base of stem close to, or with a few well above ground level, solitary; peduncle not branched, 40-80 mm long, $1-1.5 \mathrm{~mm}$ diam, densely pubescent; bracts narrowly ovate, c. 4 by $2 \mathrm{~mm}$, densely pubescent, apex blunt or acute, venation obscure. Flowers erect; pedicel and ovary c. $2.2 \mathrm{~cm}$ long, ovary c. $1.8 \mathrm{~mm}$ diam, densely pubescent; perianth dull magenta on outer surface, paler on inner surface, 3-lobed, c. $0.7 \mathrm{~cm}$ long, c. $2.5 \mathrm{~cm}$ diam, venation distinct, outer surface sparsely pubescent, inner surface sparsely puberulent; base contracted into a shallow cup, c. $0.5 \mathrm{~cm}$ deep, c. $0.8 \mathrm{~cm}$ diam, inner surface pubescent; perianth lobes deltoid, spreading to reflexed, c. 10 by $17.5 \mathrm{~mm}$, apex blunt or acute; stamens $24-30$ in 2 whorls, upper whorl with 9-14 stamens, lower whorl 15-16, filamentous; filament c. $1.5 \mathrm{~mm}$ long, glabrous; anthers positioned at the swollen connective apex, oblong, c. $1 \mathrm{~mm}$ long,

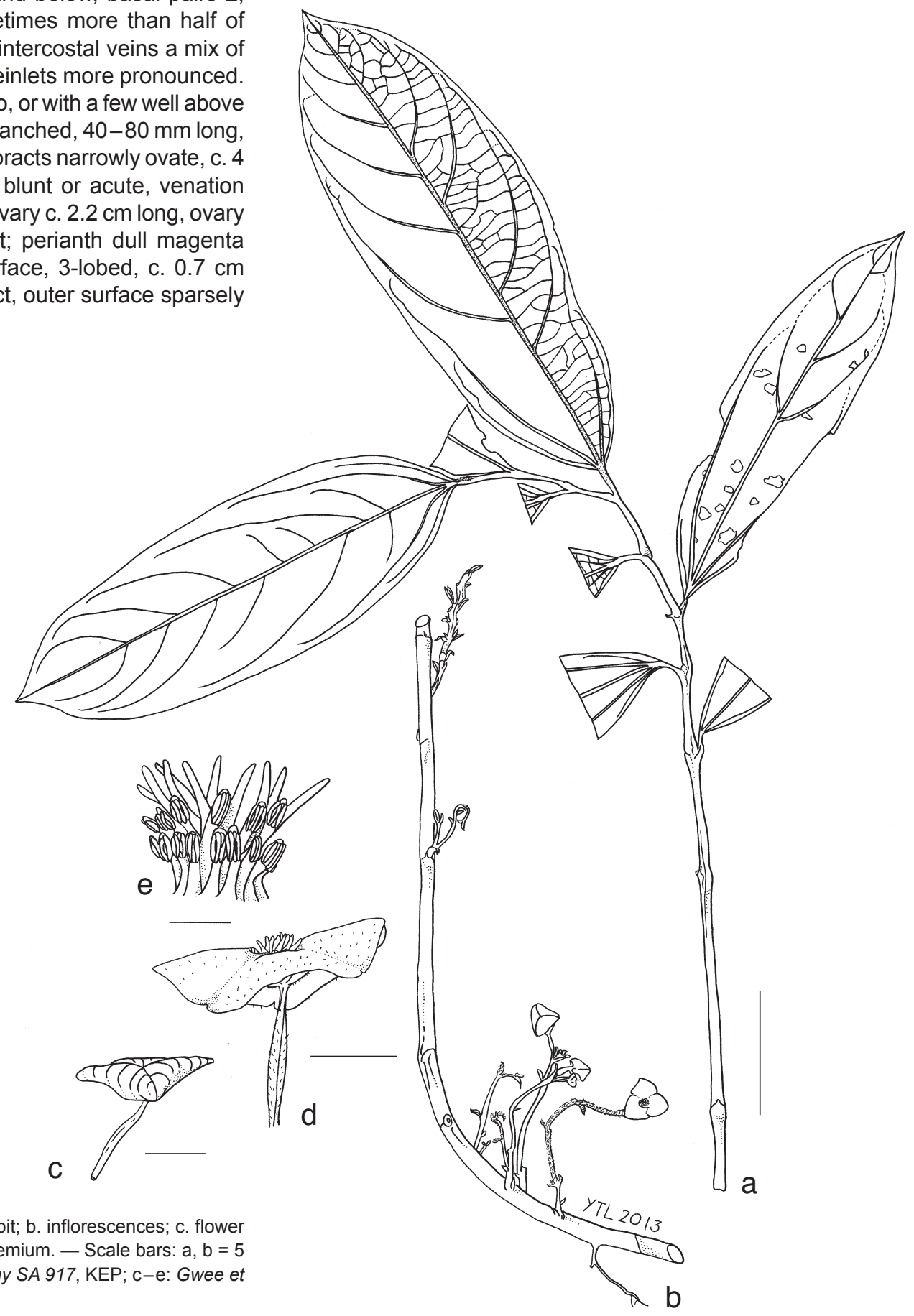

Fig. 6 Thottea praetermissa T.L.Yao. a. Habit; b. inflorescences; c. flower bud side view; $d$. flower side view; e. gynostemium. - Scale bars: $a, b=5$ $\mathrm{cm} ; \mathrm{c}, \mathrm{d}=1 \mathrm{~cm} ; \mathrm{e}=2 \mathrm{~mm}$ (a, b: Anthonysamy SA 917, KEP; c-e: Gwee et al. SING-MS 20, KEP). 
c. $0.3 \mathrm{~mm}$ wide; style column disc-shaped, c. $2.3 \mathrm{~mm}$ long, glabrous; stigmatic lobes 11-13, some lobes bifurcate, positioned higher than stamens, c. $2.6 \mathrm{~mm}$ long, glabrous. Capsules pendent, ripening brown, c. $150 \mathrm{~mm}$ long, twisted, 4-angled, c. $4 \mathrm{~mm}$ diam, densely puberulent. Seeds ovoid, 2.3-2.6 by 4-4.3 mm, 3-angled, slightly ridged, margin corrugated, testa surface pimply, apex acute, base acute or rounded.

Distribution - Peninsular Malaysia and Singapore. In Peninsular Malaysia known from Malacca and Johor. In Singapore from Western Catchment Area (extant), Sungai Morai and Sungai Buloh (historic).

Ecology - Lowland forest undergrowth, heavily shaded. On damp soil of forest close to freshwater swamp.

Conservation status (in Malaysia) - Rare. This species occurs in two sites within the network of Totally Protected Area (Chua 2012).

Additional specimens examined. Peninsular Malaysia, Johor, Gunung Ledang [Mt Ophir], Bukit Besar, Ridley s.n. (SING 97446), Dec. 1898; Mersing, Endau-Rompin State Park, Sungai Kemamuk, Yao, Pannell, Imin, Mohd Nazri FRI65617 (KEP 213072), 22 Nov. 2011; Mersing, Endau-Rompin State Park, Upeh Guling, Imin, Mohd Nazri, Kamarul Hisham FRI77804 (KEP 213073), 8 July 2012. - SINGAPORE, Sungai Morai, Goodenough 5570 (SING 3872), 28 Mar. 1893 (fl); Western Catchment, Pergam Marshes, Area D, Lee SING 2007-343 (SING 106386), 24 July 2007; Western Catchment, Pergam Marshes, Area D, Leong et al. SING 2007-367 (SING 93668; KEP 140012), 24 July 2007 (fl); Western Catchment, Pergam Marshes (near old Sungei Murai), Gwee et al. SING-MS 20 (SING 2010-831, ex-population of SING 2007-367), 22 Sept. 2010 (fl).

Notes -1 . The record of $T$. dependens from Singapore as cited in Flora of British India (Hooker 1886: 74) and followed by subsequent authors was based on two specimens, viz. Wallich 6648B (K) and Lobb $289(\mathrm{~K})$. Examination of Wallich $6648 B$ revealed that it was actually collected from Penang. The locality of Lobb's specimens is dubious (Van Steenis-Kruseman 1950: 325). Ridley (1922) pointed out that some of Lobb's specimens "... labelled 'Singapore' were undoubtedly obtained in the Himalayas, but most are Penang plants". Lobb 289 was probably also collected from Penang where $T$. dependens was common. Ridley's (1900) record of T. dependens from Sungai Buloh, Singapore, is probably based on his observation of $T$. praetermissa populations. No specimen from Sungai Buloh has been located. The first specimen of T. praetermissa was collected by Goodenough (Goodenough 5570) and was included in T. dependens by Ridley (1924) and subsequent authors. To conclude, T. dependens does not occur in Singapore.

2. For comparison of T. praetermissa, T. beccarii and T. dependens see Table 6.

\section{Thottea reflexa T.L.Yao, sp. nov. - Fig. 7, 10g; Map 1}

This species is similar to T. grandiflora in its ovate or broadly lanceolate lamina with a rounded base but it is different in its puberulent lower lamina surface (not densely pubescent as in T. grandiflora), inflorescence positioned at stem base close to ground level (not on stem well above ground level), 3-lobed perianth with bowl-shaped cupular base and completely reflexed lobes (not bell-shaped) and stamens 9-10 in one whorl (not 28-42 in two whorls). Type: Yao et al. FRI 65597 (holo KEP 192444; iso K, L), Peninsular Malaysia, Terengganu, Lata Tembakah Recreational Forest, 29 June 2011 (fl and fr).

Etymology. Latin, reflexus $=$ bend backwards at more than $90^{\circ}$; referring to the perianth lobes.

Shrub, c. $0.4 \mathrm{~m}$ tall, stem slender, erect, scarcely branched, pendent at top. Stems dark green, c. $6 \mathrm{~mm}$ diam, surface shallowly furrowed, sometimes smooth, sparsely pubescent; nodes pronounced. Leaves: bract-like reduced leaves $4-8$, foliage leaves 5-8 on one stem; petiole slender, sometimes rather stout, 8-9 mm long, c. $2 \mathrm{~mm}$ diam, pubescent; lamina green above, pale green beneath, drying brown, ovate or obovate or broadly lanceolate, $23.5-24.5$ by $11-11.5 \mathrm{~cm}$, first foliage lamina sometimes distinctly smaller, narrowly ovate, 17 by 6 $\mathrm{cm}$, leathery, indumentum beneath pubescent, base cuneate or rounded, margin entire, apex acute; midrib above flat or sometimes sunken, below prominent; venation pinnate with basal pairs, above noticeable, below prominent, basal pairs 2 , upper basal pair less than half of the leaf length, lateral pairs $6-7$; intercostal veins a mix of ladder-like and net-like, ladderlike veinlets more pronounced. Inflorescences at base of stem close to ground level, solitary or rarely clustered; peduncle sometimes once branched, 60-120 mm long, c. $1 \mathrm{~mm}$ diam, densely pubescent; bracts green, ovate, c. 4 by $2 \mathrm{~mm}$, densely pubescent, apex blunt or acute, venation obscure. Flowers pendent; pedicel and ovary c. $1.4 \mathrm{~cm}$ long, ovary c. $1.3 \mathrm{~mm}$ diam, pubescent; perianth creamy white, 3-lobed, c. $0.8 \mathrm{~cm}$ long, c. $1 \mathrm{~cm}$ diam, venation obscure, outer surface pubescent, inner surface puberulent; base contracted into a bowl-shaped cup, c. $0.5 \mathrm{~cm}$ deep, c. $0.4 \mathrm{~cm}$ diam, inner surface pubescent; perianth lobes broadly ovate, completely reflexed and blanketing perianth base, c. 6 by $9 \mathrm{~mm}$, apex retuse; stamens $9-10$ in 1 whorl, filamentous; filament c. $0.5 \mathrm{~mm}$ long, glabrous; anthers positioned at the swollen connective apex, oblong, c. $1 \mathrm{~mm}$ long, c. $0.3 \mathrm{~mm}$ wide; style column cylindric, c. $1 \mathrm{~mm}$ long, glabrous; stigmatic lobes 5-7, positioned higher than stamens, c. $1.2 \mathrm{~mm}$ long, glabrous. Capsules pendent, ripening dark purple, straight, 4-angled, c. $55 \mathrm{~mm}$ long, c. $4 \mathrm{~mm}$ diam, sparsely puberulent. Seeds ovoid, 3-angled, slightly ridged, margin corrugated, testa surface pimply, $3-3.6$ by $2.2-2.4 \mathrm{~mm}$, apex truncate, base rounded.

Table 6 Comparison of Thottea praetermissa, T. beccarii and T. dependens.

\begin{tabular}{|c|c|c|c|}
\hline & T. praetermissa & T. beccarii ${ }^{*}$ & T. dependens \\
\hline $\begin{array}{l}\text { Lamina: } \\
\text { length by width }(\mathrm{cm})\end{array}$ & $\begin{array}{l}\text { oblong or lanceolate: } \\
15-22 \text { by } 5-7.5\end{array}$ & $\begin{array}{l}\text { elliptic, rarely lanceolate or oblanceolate: } \\
20-35 \text { by } 7.5-15\end{array}$ & $\begin{array}{l}\text { ovate, obovate, oblong or oblanceolate: } \\
(17-) 19.5-28.5(-31) \text { by }(5.5-) 8.5-13(-16)\end{array}$ \\
\hline Lamina base & cuneate & cuneate or rounded & cuneate or rounded \\
\hline Lamina apex & acute, sometimes shortly mucronate & short acuminate & acute or acuminate, sometimes mucronate \\
\hline Lateral vein pairs & $5-6$ & $6-9$ & $6-8(-10)$ \\
\hline Inflorescences & at base of stem close to ground level & at base of stem & $\begin{array}{l}\text { at nodes of stem below foliage leaves well above } \\
\text { ground level }\end{array}$ \\
\hline Perianth shape & $\begin{array}{l}\text { saucer-shaped, 3-lobed; lobes deltoid, } \\
\text { spreading to reflexed }\end{array}$ & $\begin{array}{l}\text { obscurely } 3 \text {-lobed, base constricted into } \\
\text { a tube c. } 10 \mathrm{~mm} \text { long, lobes curved }\end{array}$ & $\begin{array}{l}\text { 3-lobed, base contracted into a bowl-shaped } \\
\text { cup; lobes broadly ovate, flared }\end{array}$ \\
\hline Perianth length by diam (cm) & c. 0.7 by 2.5 & $1.2-1.8$ by $2-2.5$ & $(0.8-) 1.5-2$ by $(1-) 2$ \\
\hline Stamens & $\begin{array}{l}24-30 \text { in } 2 \text { whorls; upper whorl } 9-14 \\
\text { lower whorl } 15-16\end{array}$ & $\begin{array}{l}20-24 \text { in } 2 \text { whorls; upper whorl } 7-10 \text {, } \\
\text { lower whorl } 13 \text { or } 14\end{array}$ & $\begin{array}{l}(21-) 28-30 \text { in } 2 \text { whorls; upper whorl (8-)11-12, } \\
\text { lower whorl (13-)17-18 }\end{array}$ \\
\hline Stigmatic lobe number & $11-13$ & c. 10 & $5-8$ \\
\hline
\end{tabular}

* Attributes of the characters were based on image of Beccari 528, Sumatra, Padang. Ayer Mancior, c. 360 m above sea level, August 1878 (iso K $634523, \mathrm{~K} 634524$ (http://apps.kew.org/herbcat/ getlmage.do?imageBarcode=K000634523 \& http://apps.kew.org/herbcat/getImage.do?imageBarcode=K000634524, respectively, last accessed 29 July 2013 )), and species description in Flora Malesiana (Hou 1984). 


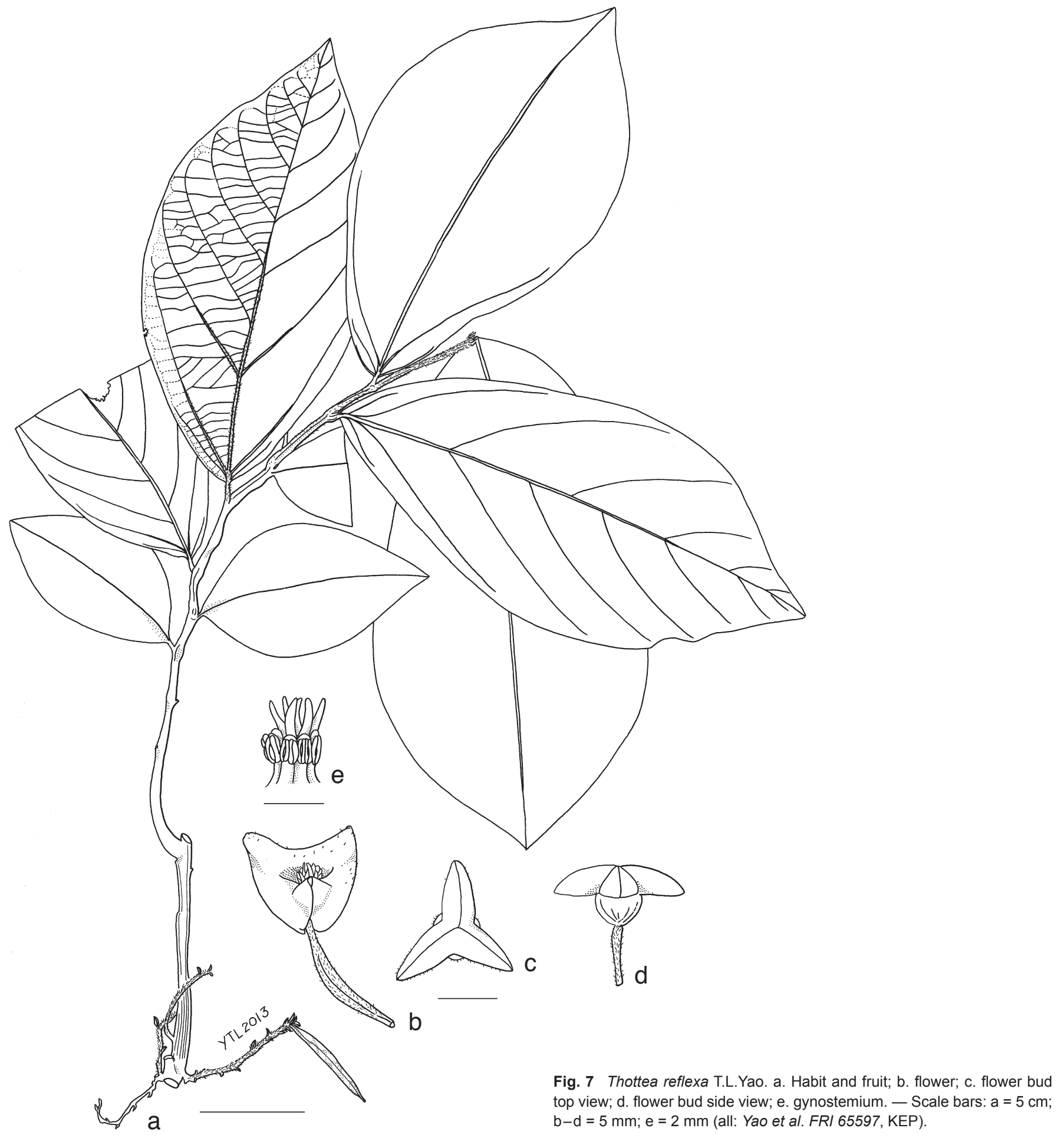

Table 7 Comparison of Thottea reflexa and T. grandiflora.

\begin{tabular}{|c|c|c|}
\hline & T. reflexa & T. grandiflora \\
\hline $\begin{array}{l}\text { Lamina: } \\
\text { length by width }(\mathrm{cm})\end{array}$ & $\begin{array}{l}\text { ovate, obovate or broadly lanceolate: } \\
23.5-24.5 \text { by } 11-11.5\end{array}$ & $\begin{array}{l}\text { ovate, oblong, broadly lanceolate or oblanceolate: } \\
(16-) 21.5-26(-43.5) \text { by }(6.5-) 10.5-24(-26.5)\end{array}$ \\
\hline Lamina under surface indumentum & puberulent & densely pubescent \\
\hline Lamina base & cuneate or rounded & cordate, sometimes rounded, rarely truncate \\
\hline Inflorescences & at base of stem close to ground level & $\begin{array}{l}\text { in axils of foliage leaf or at nodes of stem below foliage } \\
\text { leaves well above ground level }\end{array}$ \\
\hline Perianth colour & creamy white & dark claret-coloured, outside veins white \\
\hline Perianth shape & $\begin{array}{l}\text { 3-lobed, base contracted into a bowl-shaped cup; } \\
\text { lobes broadly ovate, completely reflexed and blanketing } \\
\text { perianth base }\end{array}$ & bell-shaped, 3-lobed; lobes oblong, rarely deltoid \\
\hline Perianth length by diam $(\mathrm{cm})$ & c. 0.8 by 1 & $(3-) 6-14$ by $6-10$ \\
\hline Stamens & $9-10$ in 1 whorl & $28-42$ in 2 whorls; upper whorl $12-18$, lower whorl $16-24$ \\
\hline Stigmatic lobe number & $5-7$ & $10-15$ \\
\hline Capsule indumentum & sparsely puberulent & villose \\
\hline
\end{tabular}


Distribution - Endemic to Peninsular Malaysia, known only from Lata Tembakah, Terengganu.

Ecology - Lowland forest undergrowth, on slopes or flat land, persisting in forest gaps. While collecting the type, I observed that only plants in forest gaps flower profusely.

Conservation status - Critically Endangered B2ab(iii). This species is only known from the type specimen and the type locality does not occur within the network of Totally Protected Area.

Note - For comparison of T. reflexa and T. grandiflora see Table 7.

\section{Thottea ruthiae T.L.Yao, sp. nov. - Fig. 8, 10h; Map 2}

This species is similar to $T$. sumatrana in its lanceolate lamina with rounded base, its puberulent lower lamina surface and stamens arranged in one whorl but it is different in its inflorescence position at the stem base close to ground level (not in the axils of foliage leaves as in T. sumatrana) and the greater stamen number 10 (not consistently 6). - Type: Kiew RK 2703 (holo KEP 192448, a rehydrated flower preserved in Copenhagen mixture), Peninsular Malaysia, Terengganu, Jertih, hydro-intake across river from Kampung La, 9 May 1988 (fl).
Etymology. Ruth Kiew, born 1946; a Malaysian botanist, best known for her research on Begonia (Begoniaceae) and the herbaceous and limestone flora. Project coordinator and chief editor of Flora of Peninsular Malaysia project based in KEP.

Shrub, c. $0.3 \mathrm{~m}$ tall, stem slender, erect, scarcely branched, pendent at top. Stems dark green, c. $4 \mathrm{~mm}$ diam, surface smooth, puberulent; nodes indistinct. Leaves: foliage leaves c. 6 on one stem; petiole slender, c. $10 \mathrm{~mm}$ long, c. $3 \mathrm{~mm}$ diam, densely pubescent; lamina green above, pale green beneath, drying brown, lanceolate, c. 20.5 by $8 \mathrm{~cm}$, first foliage lamina distinctly smaller, narrowly lanceolate, c. 9.5 by $4 \mathrm{~cm}$, thinly leathery, indumentum beneath pubescent, base cuneate or rounded, margin entire, apex blunt or acute; midrib above flat, below prominent; venation pinnate with basal pairs, above faint, below prominent, basal pairs 2, upper basal pair less than, or sometimes more than half of the lamina length, lateral pairs $5-7$; intercostal veins a mix of ladder-like and net-like, ladderlike veinlets more pronounced. Inflorescences at base of stem close to ground level, solitary; peduncle not branched, c. 25 $\mathrm{mm}$ long, c. $1 \mathrm{~mm}$ diam, pubescent; bracts oblanceolate, c. 1.5

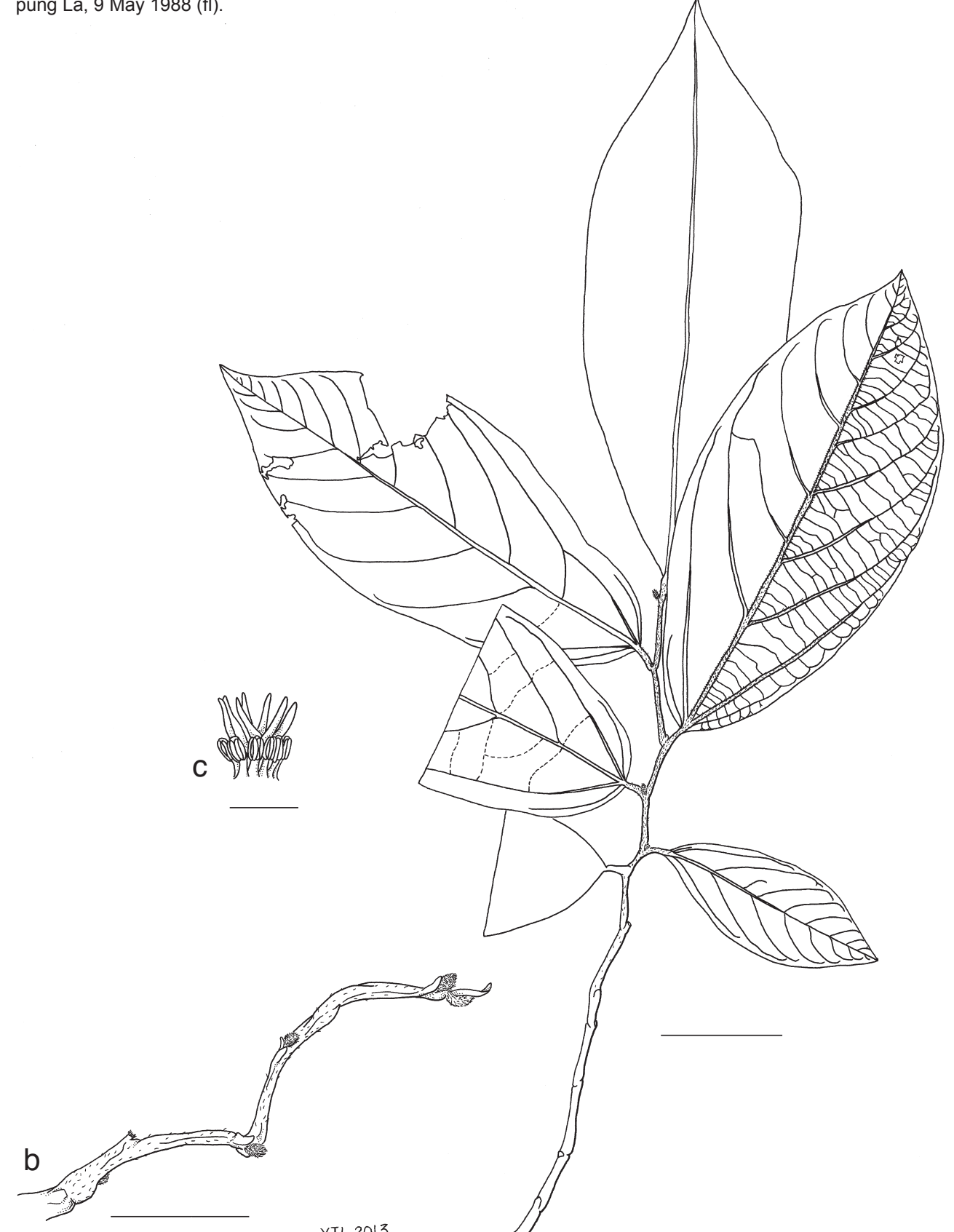

Fig. 8 Thottea ruthiae T.L.Yao. a. Habit; b. inflorescence; c. gynostemium — Scale bars: $a=5 \mathrm{~cm} ; \mathrm{b}=5 \mathrm{~mm} ; \mathrm{c}=2 \mathrm{~mm}$ (all: Kiew RK 2703, KEP). 
Table 8 Comparison of Thottea ruthiae and T. sumatrana.

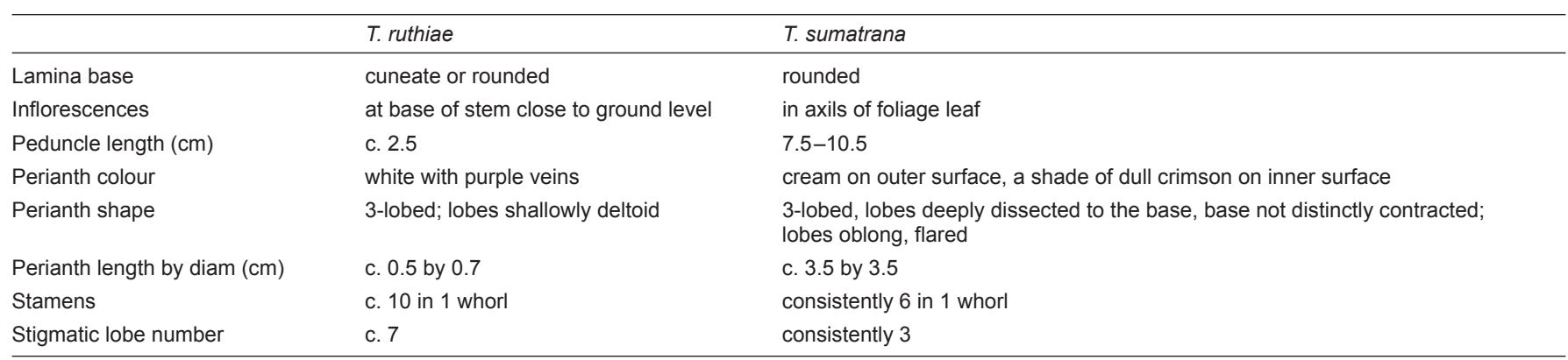

by $1 \mathrm{~mm}$, pubescent, apex blunt, venation obscure. Flowers erect; pedicel and ovary c. $0.7 \mathrm{~cm}$ long, ovary c. $1 \mathrm{~mm}$ diam, pubescent; perianth white with purple veins, 3-lobed, c. $0.5 \mathrm{~cm}$ long, c. $0.7 \mathrm{~cm}$ diam, outer surface pubescent, inner surface sparsely puberulent; perianth lobes shallowly deltoid, c. 2 by 7 $\mathrm{mm}$, apex blunt, sparsely puberulent but thinning out towards centre; stamens c. 10 in 1 whorl, filamentous; filament c. 0.3 $\mathrm{mm}$ long, glabrous; anthers positioned at the swollen connective apex, narrowly oblong, c. $0.7 \mathrm{~mm}$ long, c. $0.2 \mathrm{~mm}$ wide; style column cylindric, c. $0.7 \mathrm{~mm}$ long, glabrous; stigmatic lobes c. 7 , positioned higher than stamens, c. $0.7 \mathrm{~mm}$ long, glabrous. Capsule and seed unknown.

Distribution - Endemic to Peninsular Malaysia, known only from Kampung La, Terengganu.

Ecology - Riverine forest, common in undergrowth.

Conservation status - Critically Endangered B2ab(iii). This species has not been collected in the past 20 years. It is only known from the type specimen and the type locality does not occur within the network of Totally Protected Area.

Note - For comparison of T. ruthiae and T. sumatrana see Table 8.

9. Thottea terengganuensis T.L.Yao, sp. nov. - Fig. 9, 10i; Map 2

This species is similar to $T$. grandiflora in its broadly lanceolate lamina with rounded base, densely pubescent lower lamina surface and stamens arranged in two whorls but it is different in its inflorescences positioned at stem base close to ground level (not in axils of foliage leaf or at nodes of stem below foliage leaves well above ground level), its 3-lobed perianth with a bowl-shaped base (not bell-shaped) and its pubescent capsule (not villose). - Type: Sam \& Mohd Aidil FRI 68960 (holo KEP 192450; iso K, L), Peninsular Malaysia, Terengganu, Dungun, Jengai FR, 21 Apr. 2009 (fl).

Thottea tomentosa auct. non (Blume) Ding Hou: Hou (1984) 79, p.p.

Etymology. From Terengganu, Peninsular Malaysia, where it is locally widespread.

Shrub, 0.4-0.8 m tall, stem slender, erect, scarcely branched, pendent at top. Stems dark green, 4-6.5 mm diam, surface smooth, pubescent; nodes indistinct. Leaves: bract-like reduced leaves $2-8$, foliage leaves $(2-) 4-6$ on one stem; petiole rather stout, 4-8 $\mathrm{mm}$ long, 2-3.5 $\mathrm{mm}$ diam, densely pubescent; lamina dark green above, pale green beneath, drying brown, obovate or broadly lanceolate, $(11.5-) 19.5-30$ by $(6.5-) 12-13.5 \mathrm{~cm}$, first foliage lamina sometimes distinctly smaller, ovate, $11-16$ by $5-5.5 \mathrm{~cm}$, thinly leathery, indumentum beneath densely pubescent, base cuneate or rounded, margin entire, near base of dried specimens often in-folded, apex blunt or acute; midrib above flat, below prominent; venation pinnate with basal pairs, above faint, below prominent, basal pairs 2 , upper basal pair less than, or sometimes more than half of the lamina length, lateral pairs 5-8; intercostal veins a mix of ladder-like and net-like, ladder-like veinlets more pronounced. Inflorescences at stem base close to ground level, solitary; peduncle not branched, $8-16 \mathrm{~cm}$ long, c. $2.5 \mathrm{~mm}$ diam, pubescent; bracts green, lanceolate or ovate, $7-8$ by c. $4 \mathrm{~mm}$, densely pubescent, apex acute, venation obscure or sometimes distinct. Flowers erect or held horizontally; pedicel and ovary c. $15 \mathrm{~mm}$ long, ovary c. $1.8 \mathrm{~mm}$ diam, densely pubescent; perianth claret-coloured on outer surface, cream with claretcoloured rim (or entirely claret-coloured) on inner surface, or entirely claret, 3-lobed, c. $1 \mathrm{~cm}$ long, $3.5-5 \mathrm{~cm}$ diam, venation distinct, outer surface sparsely pubescent, inner surface puberulent; base contracted into a bowl-shaped cup, c. $0.6 \mathrm{~cm}$ deep, c. $0.8 \mathrm{~cm}$ diam, inner surface pubescent; perianth lobes shallowly deltoid, spreading, slightly laterally reflexed, c. 15 by $20-23 \mathrm{~mm}$, apex blunt; stamens $24-29$ in 2 whorls, upper whorl with 8-10 stamens, lower whorl 16-19, filamentous; filament c. $1 \mathrm{~mm}$ long, glabrous; anthers positioned at the swollen connective apex, oblong, c. $1.3 \mathrm{~mm}$ long, c. $0.6 \mathrm{~mm}$ wide; style column cylindric, $2.2 \mathrm{~mm}$ long, pubescent; stigmatic lobes 10-11, some lobes bifurcate, positioned higher than stamens, c. $2.6 \mathrm{~mm}$ long, pubescent. Capsules erect, ripening brown, straight, 4-angled, $7.3-7.5 \mathrm{~cm}$ long, c. $5.5 \mathrm{~mm}$ diam, ferruginous pubescent. Seeds ovoid, 3-angled, slightly ridged, margin corrugated, testa surface pimply, c. 3.5 by $2 \mathrm{~mm}$, apex acute, base rounded.

Distribution - Endemic to Peninsular Malaysia, known only from Terengganu.

Ecology - Lowland forest. On lateritic earth bank, occasionally by riverside. Persisting in forest gaps.

Vernacular name - Hempedu Beruang (Malay).

Conservation status - Vulnerable B1ab(iii). This species is confined to a single state and no population occurs in the network of Totally Protected Areas.

Additional specimens examined. Peninsular Malaysia, Terengganu, 34th mile Kuala Terengganu-Besut Road (east side), Sinclair \& Kiah SFN 40768 (SING 97441), 8 Sept. 1955; Bukit Bauk FR, Zainudin AZ 2716 (UKMB), 21 Mar. 1989; Kuala Berang, Sungai Berua, indigenous community village, Zainudin \& Wan Faizah AZ 4680 (UKMB 24588), 11 Sept. 1993; Sekayu Recreational Forest, trail between chalet and river, Yao et al. FRI 65444 (KEP 192696), 8 Apr. 2009 (fl); Dungun, Jerangau FR, buffer zone between compartment 35 and state land, Lim \& Mohd. Hairul FRI 65102 (KEP 192697), 24 July 2009 (fl); Dungun, Pasir Raja FR, Compartment 5, Yao \& Azril FRI 65589 and FRI 65591 (KEP 192698 and 192699, resp.), 28 June 2011 (fl and fr).

Notes - 1. The margin near lamina base of dried specimens is often revolute.

2. There is variation in flower colour. I observed that the Pasir Raja FR population has entirely claret-coloured inner surface perianth in contrast to cream with claret-coloured rim.

3. At Bukit Bauk, it is sympatric with T. grandiflora. Both species look similar when not in flower but $T$. terengganuensis is distinctive in its more prominent tertiary veins and smaller leaves.

4. Small-leaved specimens of $T$. terengganuensis superficially resemble $T$. tomentosa specimens but leaf number per stem is a good character in distinguishing them $-T$. terengganuensis has more than four leaves while $T$. tomentosa usually has up to three.

5. For comparison of T. terengganuensis and T. grandiflora see Table 9 . 
Table 9 Comparison of Thottea terengganuensis and T. grandiflora.

\begin{tabular}{|c|c|c|}
\hline & T. terengganuensis & T. grandiflora \\
\hline $\begin{array}{l}\text { Lamina: } \\
\text { length by width }(\mathrm{cm})\end{array}$ & $\begin{array}{l}\text { obovate or broadly lanceolate: } \\
(11.5-) 19.5-30 \text { by }(6.5-) 12-13.5\end{array}$ & $\begin{array}{l}\text { ovate, oblong, broadly lanceolate or oblanceolate: } \\
(16-) 21.5-26(-43.5) \text { by }(6.5-) 10.5-24(-26.5)\end{array}$ \\
\hline Lamina base & cuneate or rounded & cordate, sometimes rounded, rarely truncate \\
\hline Inflorescences & at base of stem close to ground level & $\begin{array}{l}\text { at axils of foliage leaf or at nodes of stem below foliage leaves } \\
\text { well above ground level }\end{array}$ \\
\hline Perianth colour & $\begin{array}{l}\text { claret-coloured outside, cream with claret-coloured rim on } \\
\text { inner surface, or entirely claret }\end{array}$ & dark claret-coloured, outside veins white \\
\hline Perianth shape & $\begin{array}{l}\text { 3-lobed, base contracted into a bowl-shaped cup; shallowly } \\
\text { deltoid, spreading }\end{array}$ & bell-shaped, 3-lobed; lobes oblong, rarely deltoid \\
\hline Perianth length by diam $(\mathrm{cm})$ & c. 1 by $3.5-5$ & $(3-) 6-14$ by $6-10$ \\
\hline Stamens & $24-29$ in 2 whorls; upper whorl $8-10$, lower whorl $16-19$ & $28-42$ in 2 whorls; upper whorl $12-18$, lower whorl $16-24$ \\
\hline Stigmatic lobes number & $10-11$ & $10-15$ \\
\hline Capsule indumentum & pubescent & villose \\
\hline
\end{tabular}

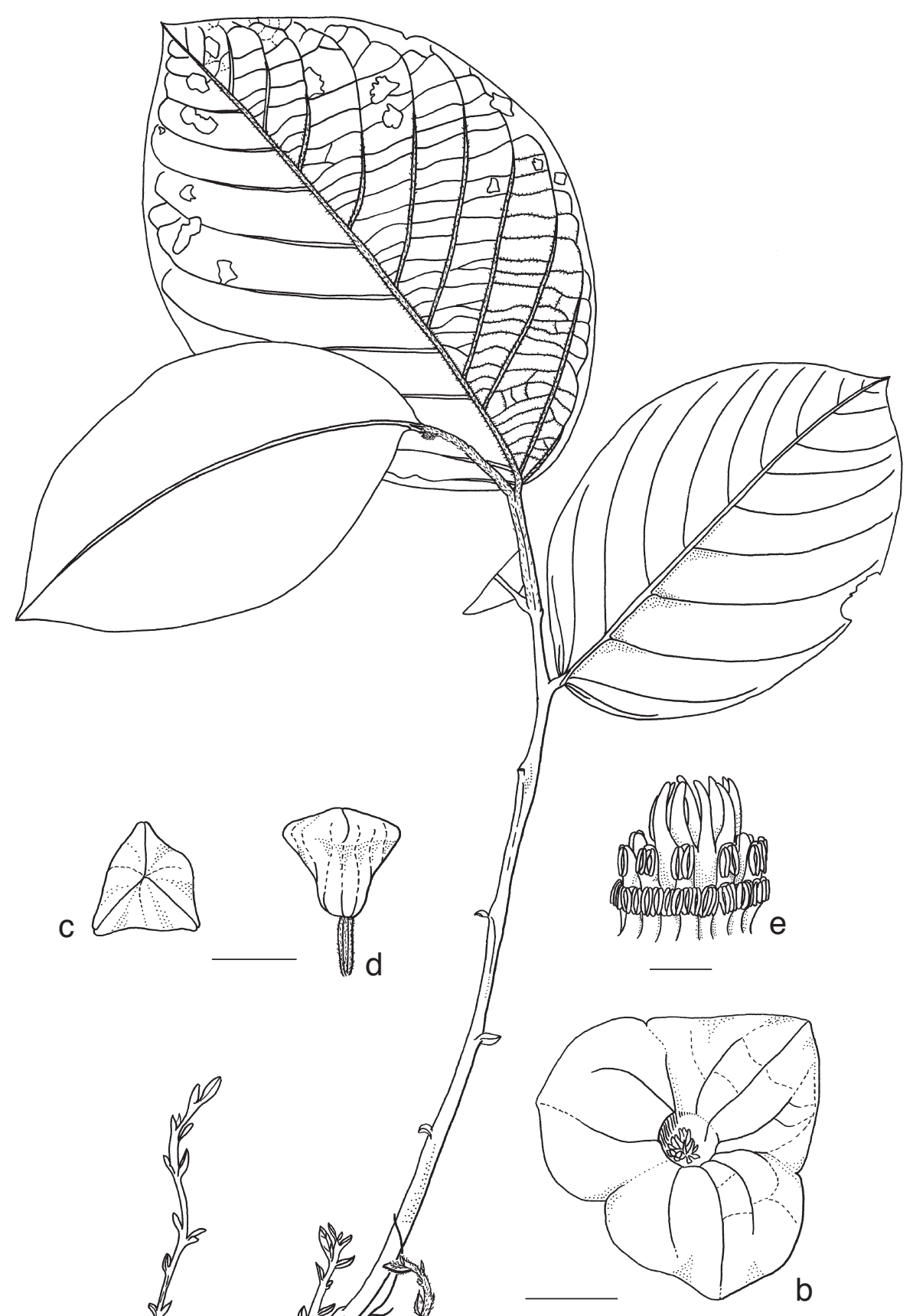

Fig. 9 Thottea terengganuensis T.L.Yao. a. Habit and inflorescences; b. flower; c. flower bud top view; d. flower bud lateral view; e. gynostemium. - Scale bars: $a=5 \mathrm{~cm} ; b-d=1 \mathrm{~cm} ; \mathrm{e}=2 \mathrm{~mm}$ (a, c-e: Sam \& Mohd Aidil FRI 68960, KEP; b: Yao et al. FRI 65444, KEP). 


\section{REDEFINED TAXA}

\section{Thottea dependens (Planch.) Klotzsch - Map 3}

Thottea dependens (Planch.) Klotzsch (1859) 589; Duch. (1864) 428; Hook.f. (1886) 74; Ridl. (1900) 127; Gamble (1912) 28; Ridl. (1924) 16; Ding Hou (1981) 311, f. 5-7; (1984) 75, f. 1 b.

Lobbia dependens Planch. (1847) 144; Miq. (1858) 1068. - Type: Lobb 289 (holo K 575922; iso BM 950665, K 575923, 575924), 'Singapore' (see notes under $T$. praetermissa), 1846.

Piper arborescens Roxb. ex Wall. (1832) 224. - Type: Wallich 6648B (holo K 575918), Peninsular Malaysia, Penang, 1822.

Etymology. Latin, dependeo = to hang down; referring to the inflorescence.

Shrub, 0.5-2 m tall, stem slender, erect, scarcely branched, pendent at top. Stems dark green, 4-8 mm diam, surface smooth, sparsely puberulent; nodes swollen, above nodes constricted, nodes with repeated flowering thickened. Leaves: bract-like reduced leaves c. 4 , foliage leaves $6-10$ on one stem; petiole stout, 10-18 mm long, 2-3 mm diam, puberulent; lamina pale green beneath, sometimes slightly glaucous or glaucous or drying brown, ovate, obovate, oblong or oblanceolate, (17-) $19.5-28.5(-31)$ by $(5.5-) 8.5-13(-16) \mathrm{cm}$, first foliage lamina distinctly smaller, narrowly ovate, c. 4.5 by c. $1.5 \mathrm{~cm}$, thinly leathery, indumentum beneath puberulent, base cuneate or rounded, margin entire, apex acute, acuminate, shortly mucronate or mucronate; midrib above slightly raised, below prominent; venation pinnate with basal pairs, above obscure, below distinct, basal pairs 2, upper basal pair less than, or sometimes more than half of the lamina length, lateral pairs $6-8(-10)$; intercostal veins a mix of ladder-like and net-like, ladder-like veinlets more pronounced, net-like veinlets obscure. Inflorescences on stem below foliage leaves, well above ground level, solitary; peduncle branched, 1-2 time(s), 28-45(-60) mm long, 1-2 $\mathrm{mm}$ diam, puberulent or pubescent; bracts green, narrowly oblanceolate or narrowly ovate, (3-)6-10 by (1-)2-4 $\mathrm{mm}$,

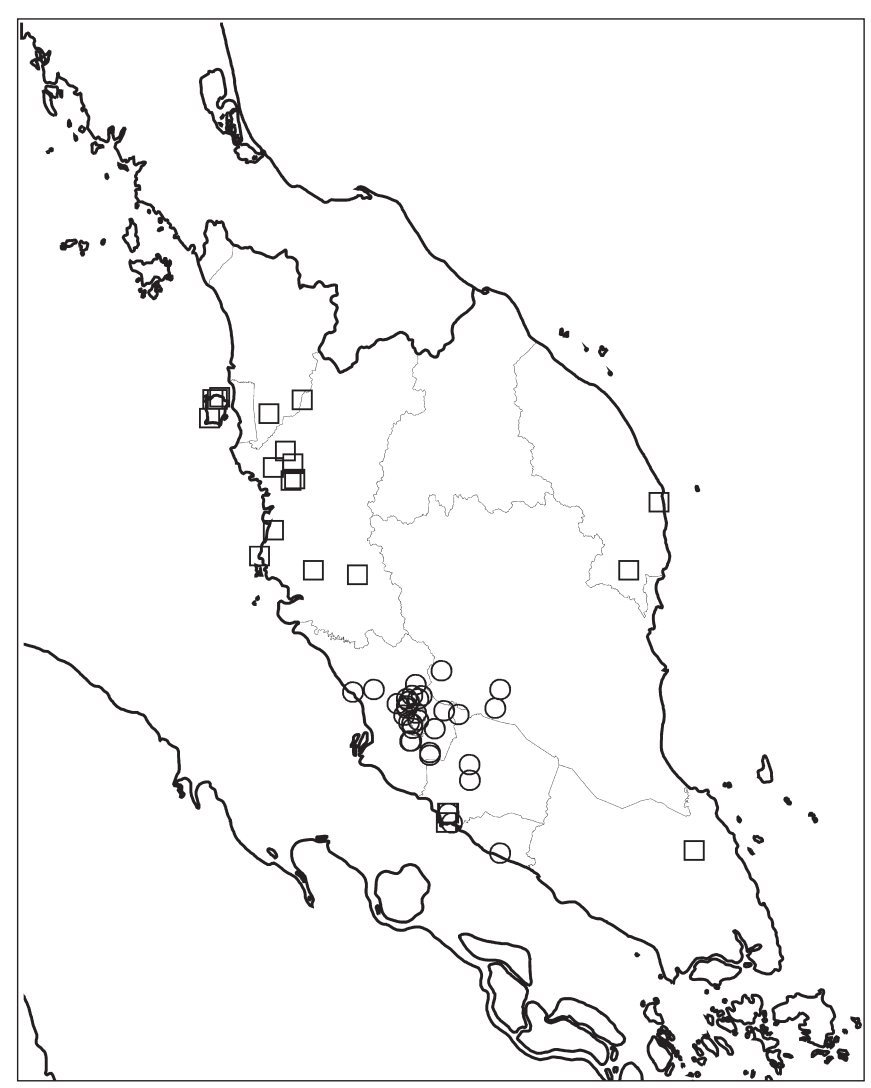

Map 3 Distribution of Thottea dependens ( $\square$ ) and T. tricornis ( $\circ$ ) in Peninsular Malaysia. Note that both species are sympatric in Sungai Menyala Forest Reserve, Negeri Sembilan. pubescent, apex acute, venation obscure or sometimes distinct. Flowers pendent; pedicel and ovary $1-2.4 \mathrm{~cm}$ long, ovary 0.5-1 mm diam, pubescent; perianth dark magenta throughout, 3-lobed, (0.8-)1.5-2 cm long, (1-)2 cm diam, venation faint, outer surface puberulent or pubescent, inner surface puberulent; base contracted into a bowl-shaped cup, (0.4-)0.7-1 $\mathrm{cm}$ deep, (0.8-)0.9-1.5 cm diam, inner surface sparsely puberulent; perianth lobes broadly ovate, flared, (4-)6-10 by 9-13 mm, apex acute; stamens $23-30$ in 2 whorls, upper whorl with (8-)11-12 stamens, lower whorl (13-)17-18, filamentous; filament c. $2 \mathrm{~mm}$ long, glabrous; anthers positioned at the swollen connective apex, oblong, c. $1.8 \mathrm{~mm}$ long, c. 0.4 $\mathrm{mm}$ wide; style column cylindric, c. $2 \mathrm{~mm}$ long, glabrous; stigmatic lobes 5-8, positioned higher than stamens, c. $2 \mathrm{~mm}$ long, glabrous. Capsules pendent, ripening brown, straight to slightly twisted, 4-angled, 130-170 mm long, 3-10 mm diam, puberulent. Seeds ellipsoid, 3-angled, slightly ridged, margin corrugated, testa surface transversely strongly wrinkled, 4-5 by $1.5-2 \mathrm{~mm}$, apex and base acute.

Distribution - Endemic to Peninsular Malaysia, known from Kedah, Penang, Perak, Negeri Sembilan, Terengganu and Johor.

Ecology - 1. Lowland and coastal hill forests. On lateritic ridges and slopes or sometimes on sandy soil, humus layer thin.

2. The fully ripe capsule splits from top to the base exposing a string of seeds covered in white glistening starchy pulp hanging on to the funicles. The dispersal mechanism inferred from this observation as being the white worm-like string of seeds is to attract ground foraging birds to consume the seeds.

Vernacular name - Telinga beruang (Malay).

Conservation status - Least Concern. This species is widespread and locally common.

\section{Thottea tricornis Maingay ex Hook.f. - Map 3}

Thottea tricornis Maingay ex Hook.f. (1886) 74; Gamble (1912) 29; Ridl. (1924) 16; Ding Hou (1981) 318, f. 2, 17, 18, 43, 44, 61, 70B; (1984) 74, f. 7f-i. - Type: Maingay 1819 (=Kew Distr. No. 1319) (holo K 575919; iso BM 950668, K 575920, 575921, L 38825), Peninsular Malaysia, Malacca, Aug. 1866

Etymology. Latin, tri- = three, cornis = horn-shape; referring to the 3-cornered flower buds.

Shrub, 0.65-1.8 m tall, stem stout, erect, scarcely branched, pendent at top. Stems dark green, 4-9 mm diam, surface smooth, sparsely puberulent or glabrescent; nodes pronounced. Leaves: bract-like reduced leaves $4-5$, foliage leaves $7-15$ on one stem; petiole stout, $10-15 \mathrm{~mm}$ long, $2.5-4 \mathrm{~mm}$ diam, puberulent; lamina green above, pale green beneath, drying brown, broadly ovate, narrowly oblanceolate or oblanceolate, (16.5-)22-36.5 by $8-16.5 \mathrm{~cm}$, first foliage lamina distinctly smaller, oblong, c. 6 by $2 \mathrm{~cm}$, papery to thinly leathery, indumentum beneath puberulent, base cuneate, margin entire, apex acute or acuminate; midrib above distinct, below prominent; venation pinnateacrodromous, above distinct, below prominent, basal pairs 2, upper basal pair more than half of the lamina length, lateral pairs 4-8; intercostal veins a mix of ladder-like and net-like, ladderlike veinlets more pronounced. Inflorescences in axils of foliage leaf, solitary; peduncle not branched, 30-60 mm long, c. 1.5 $\mathrm{mm}$ diam, densely pubescent or glabrescent; bracts light green, narrowly lanceolate or narrowly oblanceolate, $5-11$ by $2-5 \mathrm{~mm}$, densely pubescent, apex acute, venation obscure, sometimes distinct. Flowers pendent; pedicel and ovary c. $2 \mathrm{~cm}$ long, ovary c. $1 \mathrm{~mm}$ diam, pubescent; perianth magenta on outer surface, creamy white on inner surface, 3-lobed, funnel-shaped, base not distinctly contracted, $1.4-1.5 \mathrm{~cm}$ long, $1.5-3.5 \mathrm{~cm}$ diam, venation faint, outer surface sparsely pubescent, inner surface 
pubescent; perianth lobes faintly deltoid, flared, c. 20 by $2 \mathrm{~mm}$, apex acute or mucronate; stamens $23-26$ in 2 whorls, upper whorl with 9-10 stamens, lower whorl 14-16, filamentous; filament c. $0.5 \mathrm{~mm}$ long, glabrous; anthers at the swollen apical tip of the filament, oblong, c. $1.2 \mathrm{~mm}$ long, c. $0.4 \mathrm{~mm}$ wide; style column disc-shaped, c. $2 \mathrm{~mm}$ long, glabrous; stigmatic lobes 8-13, positioned higher than stamens, 1-2 mm long, glabrous. Capsules pendent, ripening brown, straight to slightly twisted, 4-angled, 95-140 mm long, 4-7 mm diam, densely puberulent. Seeds ovoid, 3-cornered, margin corrugated, testa surface pimply, c. 6 by $2.5 \mathrm{~mm}$, acute at both apex and base.

Distribution - Endemic to Peninsular Malaysia, known from Perak, Selangor, Negeri Sembilan, Malacca and Pahang. Common and widespread in Selangor but scattered in others states.

Ecology - Usually in lowland to hill forests to $760 \mathrm{~m}$ altitude, rarely in coastal hill forest. On lateritic earth bank and occasionally by sandy streamside. Persisting in secondary forest and old rubber estates. Kiew (1984) refers to this species. Caterpillar of Golden Birdwing Butterfly feed on this species (Kiew 2010).

Vernacularnames - Melada, Telinga kelawar (Malay); Chengrus, Geram rengkong, Pokok teringkum, Tusa lanjut (Temuan); Chudok (? Temuan/Jakun).

Conservation status - Least Concern. This species is widespread, locally common and persists in old secondary forests.

\section{LECTOTYPIFIED TAXON}

\section{Thottea parviflora Ridl.}

Thottea parviflora Ridl. (1910) 89; (1924) 17; Ding Hou (1981) 305, f. 38-40, 70D; (1984) 67, f. 7n-p; Phuph. (1987) 28, f. 18. - Type: Ridley 14580 (lecto SING 97415, designated here; isolecto BM 950667, K 634535), Peninsular Malaysia, Perak, Temenggor [Temango], July 1909.

I designated Ridley 14580 as the lectotype because it is the only collection antedating the protologue and it was collected by the author himself.

\section{CONCLUSIONS}

\section{Novelties and new characters}

These novelties bring the species number of Thottea species in Peninsular Malaysia to 16. The discovery of them also revealed some new characters in Peninsular Malaysian Thottea. Creamy white flowers are observed in T. anthonysamyi, T. piscodora and T. reflexa. Bicoloured flowers are observed in T. papilionis where the deep claret-coloured perianth contrast with the creamy white patch on each perianth lobe, and in $T$. terengganuensis with claret on outer surface and claret-coloured rimmed creamy white inner surface. Entirely claret-coloured flowers are also observed in T. terengganuensis. Variable flower colouration is not rare in Thottea species. Thottea tomentosa also has both deep claret-coloured and yellowish cream flowers. Bicoloured flowers and variable flower colouration are also observed in taxa of Western Ghats, India (Shaiju \& Omanakumari 2010).
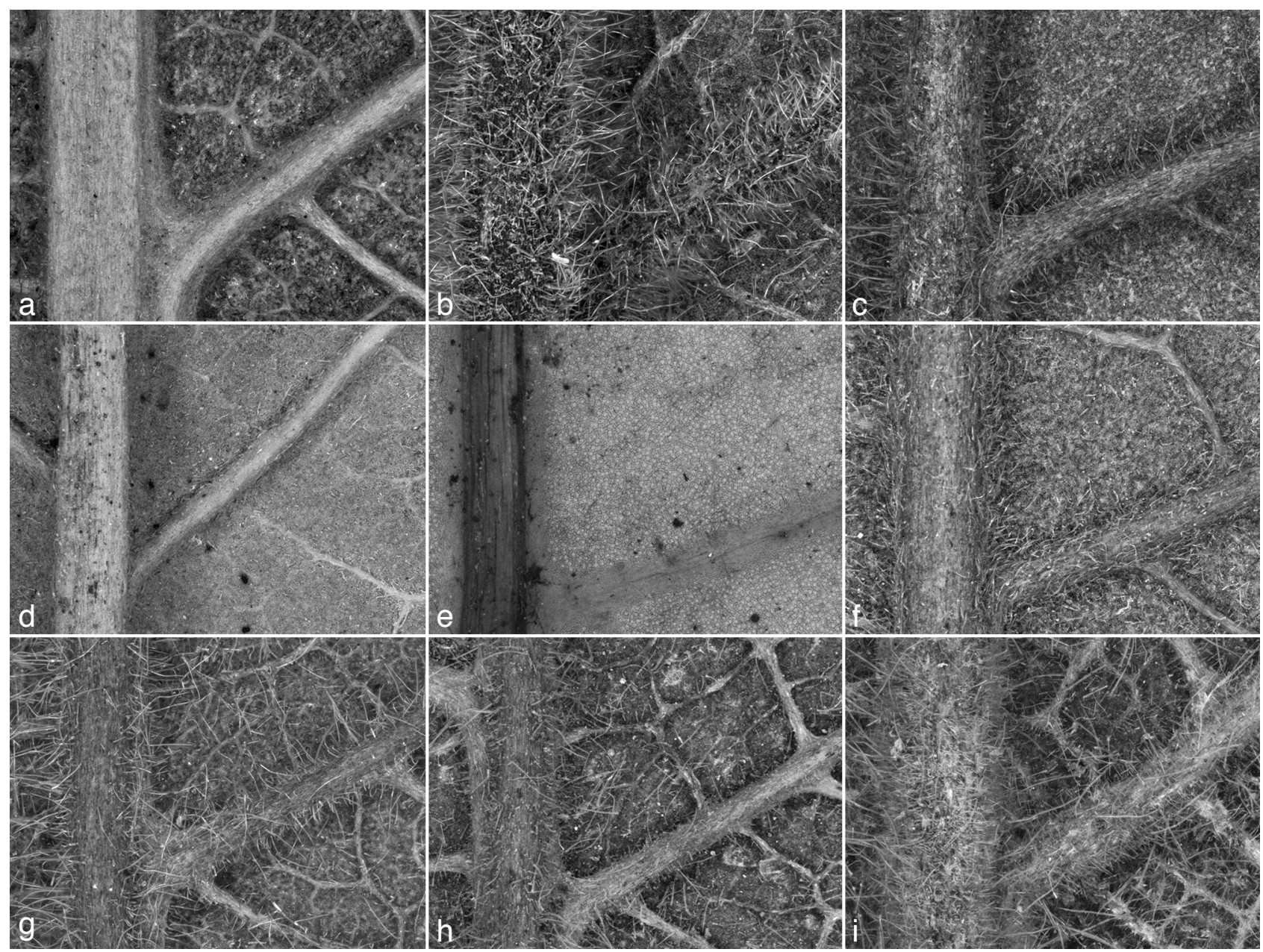

Fig. 10 Indumentum of lamina lower surface under 20x magnification. a. Thottea anthonysamyii; b. T. kamarudiniana; c. T. longipedunculata; d. T. papilionis; e. T. piscodora; f. T. praetermissa; g. T. reflexa; h. T. ruthiea; i. T. terengganuensis (a: Anthonysamy s.n., SING 15183; b: Sani et al. SM 449, UKMB; c: Motan KEP 94538, KEP; d: Yao et al. FRI 65421, KEP; e: Yao et al. FRI 65443, KEP; f: Anthonysamy SA 917, KEP; g: Yao et al. FRI 65597, KEP; h: Kiew RK 2703, KEP; i: Yao \& Azril FRI 65589, KEP). 
The spherical-ovoid perianth of $T$. anthonysamyi is observed for the first time in Thottea species.

The strong rotten fish smell of $T$. piscodora flowers makes it unique among Thottea species. Most Thottea species have scentless flowers although some of them have the dull red colour of 'carrion flowers' (Polunin 2004), and flies were often observed visiting flowers of $T$. tomentosa (pers. obs.).

Among Thottea species, the indumentum of the lower surface of the lamina (see Fig. 10), and the position of the inflorescence are consistent taxonomic characters. Peduncle length does separate some species either those with very long peduncles, viz. T. longipedunculata (c. $28 \mathrm{~cm}$ ) or very short peduncles, viz. T. ruthiea (c. $2.5 \mathrm{~cm}$ ). However, peduncle length in some species, especially those with inflorescences positioned close to ground level, are quite variable, e.g. $4-8(-25) \mathrm{cm}$ in T. tomentosa, $8-16 \mathrm{~cm}$ in T. terengganuensis and $6-12 \mathrm{~cm}$ in T. reflexa.

The structure of the flower is a crucial specific character. Stamen number of $T$. tomentosa and $T$. sumatrana is consistently 6 but in other species can be quite variable, e.g. 24-30 in T. praetermissa and 28-42 in T. grandiflora. The number of stamen whorls is, however, consistent within a species. Among Peninsular Malaysian species, T. piperiformis, T. tomentosa, $T$. ruthiae, $T$. reflexa and $T$. sumatrana have up to 10 stamens arranged in one whorl, while the rest have 2 whorls. (It is not known for T. kamarudiniana). Ridley (1924) and Hou (1984) recognised 4 whorls of stamens in T. parviflora. My study of a mature flower of $T$. parviflora found the stamens are arranged in two complete whorls with a few others scattered but are not forming a whorl.

The characters of the capsule do not provide much information for species identification. However, the villose capsules of T. grandiflora and T. kamarudiniana separate them from the rest.

\section{Conservation status}

All novelties except $T$. praetermissa and $T$. terengganuensis have very restricted distributions, many confined to one or two localities. Notably, six out of nine novelties are confined to the east coast of Peninsular Malaysia. IUCN Conservation Status of eight novelties falls in threatened categories of which six are Critically Endangered (Table 10). Thottea praetermissa is the only new species with populations occurring within Totally Protected Areas, namely Gunung Ledang State Park and Endau-Rompin State Park, while the habitat of the rest is not protected by legislation related to Totally Protected Areas.

Acknowledgements This article is dedicated to Ding Hou (1921-2008), who laid concrete taxonomic foundations for Malesian Aristolochiaceae. The present study is part of the 'Flora of Peninsular Malaysia project' (01-04-010000 Khas) and 'Documentation \& Inventory Flora of Malaysia Project' based at Forest Research Institute Malaysia and fully funded by the Ministry of Science, Technology and Innovation, Malaysia (MOSTI) and 10th Malaysian Plan (RMKe-10), respectively. Curators, keepers and managers of herbaria (BM, K, KLU, L, SING, SINU, UKMB) are gratefully acknowledged for allowing me to examine or loan the specimens in their care. I thank R. Kiew, L.G. Saw and R.C.K. Chung (KEP) for their advice and comments on the manuscript. I am also grateful to R. Kiew for her interest on this genus and

Table 10 Conservation status category of the new Thottea species.

\begin{tabular}{ll}
\hline Category and criteria & Species \\
\hline Critically Endangered B1ab(iii) & Thottea longipedunculata \\
Critically Endangered B2ab(iii) & $\begin{array}{l}\text { Thottea kamarudiniana, T. papilionis, } \\
\text { T. piscodora, T. reflexa, T. ruthiae }\end{array}$ \\
Endangered B2ab(iii) & Thottea anthonysamyi \\
Rare & Thottea praetermissa \\
Vulnerable B1ab(iii) & Thottea terengganuensis \\
\hline
\end{tabular}

valuable advice throughout my study. J.F. Veldkamp (L) is acknowledged for his advice and suggestions on species naming. I thank Forestry Department of Peninsular Malaysia, State Forestry Departments for granting me permission to carry out field work in the forest reserves under their authority. S. Anthonysamy is gratefully acknowledged for guiding in the field. I thank P.K.F. Leong (SING) for sending me the flowers of Singapore specimens in spirit. M. Sani (UKMB) communicated the exact locality of T. kamarudiniana; Wan Husin, Forest Ranger of Terengganu Forestry Department provided the map of Pasir Raja Forest Reserve and S. Ismail of Terengganu Forestry pointed me to flowering individuals of $T$. reflexa in Lata Tembakah Recreational Forest. They are all gratefully acknowledged for their helpfulness. I am indebted to the KEP team: A. Angan, A. Azril, M.A. Mohd Hairul, A. Mohd Nazri and A. Norzamli for their competent assistance in the field. I thank P.T. Ong and J.P.C. Tan (KEP) for taking care of the living collections in the nursery. Last but not least, I would like to thank the anonymous reviewers and editor for improving the manuscript.

\section{REFERENCES}

Beccari O. 1870. Illustrazione di Nuove Specie di Piante Bornensi. Nuovo Giornale Botanico Italiano 2: 5-8.

Chua LSL. 2012. Conservation. In: Kiew R, Chung RCK, Saw LG, Soepadmo E (eds), Flora of Peninsular Malaysia, Ser. II, 3: 3-10. Malayan Forest Record 49, Forest Research Institute Malaysia, Kepong, Malaysia.

Duchartre P. 1864. Aristolochiaceae. In: De Candolle AP, Prodromus systematis naturalis regni vegetabilis 15, 1: 421-498. Sumptibus Sociorum Treuttel \& Würtz, Paris, France.

Faridah-Hanum I, Khamis S, Manap TA, Suterisno SE, Latifah ZA, Mohd Nasir Mohamad, Ibrahim AZ, Latiff A. 2006. An annotated checklist of seed plants at Pasir Raja Forest Reserve. In: Azahar M, Na'aman J, Mohd Rizal S, Jalil Md S, Nizam MS, Latiff A (eds), Siri Kepelbagaian Biologi Hutan 7: Gunung Mandi Angin, Terengganu. Pengurusan, Persekitaran Fizikal, Kepelbagaian Biologi dan Pelancongan Ekologi: 114-154. Jabatan Perhutanan Semenanjung Malaysia, Kuala Lumpur, Malaysia.

Gamble JS. 1912. Aristolochiaceae. Flora of Malayan Peninsula. Journal of the Asiatic Society of Bengal 75: 23-33.

Hooker JD. 1886. Aristolochiaceae. The Flora of British India, 5: 72-77. Reeve \& Co. Ltd., Kent, UK.

Hou D. 1981. Florae Malesianae Praecursores LXII. On the genus Thottea (Aristolochiaceae). Blumea 27: 301-332.

Hou D. 1983. Florae Malesianae Praecursores LXIII. New species of Malesian Aristolochiaceae. Blumea 28: 343-352.

Hou D. 1984. Aristolochiaceae. Flora Malesiana, Ser. I, 10: 53-108.

Kiew R. 1984. Notes on the natural history of Thottea dependens (Aristolochiaceae) and the occurrence of foliar sclereids within the genus. The Malayan Nature Journal 38: 59-64.

Kiew R. 2010. Bukit Gasing's hidden treasure: The golden birdwing butterfly. Malaysian Naturalist 63: 10-11.

Kiew R, Chung RCK, Saw LG, Soepadmo E. 2010. Seed plant families in Peninsular Malaysia. In: Kiew R, Chung RCK, Saw LG, Soepadmo E (eds), Flora of Peninsular Malaysia, Ser. II, 1: 3-20. Malayan Forest Record 49, Forest Research Institute Malaysia, Kepong, Malaysia.

Kiew R, Rafidah AR. 2007. The flora of Peninsular Malaysia. Conservation Malaysia Bulletin 5: 1-4.

Klotzsch JF. 1859. Die Aristolochiaceae des Berliner Herbariums. Monatsberichte der Königlichen Preussische Akademie der Wissenschaften zu Berlin: 571-626.

Miquel FAW. 1858. Aristolochieae. Flora van Nederlandsch Indië, 1. 10651069.

Oelschlägel B, Wagner S, Salomo K, Pradeep NS, Yao TL, et al. 2011. Implications from molecular phylogenetic data for systematics, biogeography and growth form evolution of Thottea (Aristolochiaceae). The Gardens' Bulletin Singapore 63: 259-275.

Phuphathanaphong L. 1987. Aristolochiaceae. In: Smitinand T, Larsen K (eds), Flora of Thailand, 5: 1-31. The Forest Herbarium, Royal Forest Department, Bangkok, Thailand.

Planchon JE. 1847. Observations sur l'Amoreuxia, DC. (Euryanthe, Cham. et Schlecht.) et descriptions des nouveaux genres Roucheria et Lobbia: comme introduction à des mémoires distincts sur les Cochlospermées, Linées et Aristolochiées, familles auxquelles ces genres seront respectivement rattachés. Hooker's London Journal of Botany 6: 139-146.

Polunin I. 2004. Plants and flowers of Malaysia. Times Editions, Singapore. Ridley HN. 1900. The Flora of Singapore. Journal of the Straits Branch of the Royal Asiatic Society 33: 27-196.

Ridley HN. 1910. A scientific expedition to Temengoh, Upper Perak. Journal of the Straits Branch of the Royal Asiatic Society 57: 5-122. 
Ridley HN. 1922. Introduction. The Flora of Malay Peninsula 1: ix-xx. Reeve \& Co. Ltd., London, UK.

Ridley HN. 1924. Aristolochiaceae. The Flora of Malay Peninsula 3: 14-19. Reeve \& Co. Ltd., London, UK.

Shaiju PN, Omanakumari N. 2009. Inflorescence morphology and systematics of the genus Thottea Rottb. (Aristolochiaceae) from the Western Ghats, India. Systematics and Biodiversity 7: 445-451.

Shaiju PN, Omanakumari N. 2010. Floral morphology and systematics of the genus Thottea Rottb. (Aristolochiaceae) from the Western Ghats, India. Plant Systematic and Evolution 288: 213-225.

Van Steenis-Kruseman MJ. 1950. Illustrated alphabetical list of the collectors. Flora Malesiana, Ser. I, 1: 5-598.

Wallich N. 1832. A numerical list of dried specimens of plants in the East India Company's Museum: collected under the superintendence of Dr. Wallich of the Company's botanic garden at Calcutta, no. 6225-7283.

\section{IDENTIFICATION LIST OF SPECIMENS CONSULTED FOR COMPARISON}

Thottea dependens: Peninsular Malaysia, Anthonysamy SA 880 (KEP); Burkill 9027 (SING); 13227 (K, SING); Corner s.n. (SING 97360, 97372); Curtis 1170 (K, SING), 1507 (K, SING), s.n. (SING 97365, 97366, 97370); Imin et al. FRI 74657 (KEP); Ishak FMS 11304 (KEP); Mohd Haniff 10557 (SING), 21113 (SING); Mohd Haniff \& Mohd Nur 3407 (SING); Ng FRI 27375 (KEP); Ong et al. FRI 71444 (KEP); Poore 5063 (KLU); Ridley 10346 (K, SING), 14042 (K, SING), s.n. (SING 97362, 97367, 97377); Sam et al. FRI 47028 (KEP); Sidek S 295 (K, SING); Wray Jr. 1318 (K); Wyatt-Smith KEP 78650 (KEP); Yao et al. FRI 65471 (KEP).

Thottea grandiflora: PenINSULAR MALAYSIA, Addison SFN 36993 (SING); Alvins 2107 (SING), s.n. (SING 97410, 97411); Anthonysamy SA 875 (KEP), SA 918 (KEP); Baharudin BB 76 (UKMB); Burkill \& Mohd Haniff 15708 (SING); Cantley's collector s.n. (SING 97408); Chan FRI 23854 (KEP); Chew FRI 60297 (KEP); Chew et al. FRI 53714 (KEP), FRI 60115 (KEP); Chin \& Mustafa 3340 (K, SING); Corner s.n. (SING 97385, 97400, 97407, 97409); Curtis 3662 (SING); Derry 126 (SING); Durant FMS 26626 (KEP); Goodenough 1343 (SING), 1473 (SING); Griffith 4439 (K); Henderson SFN 24102 (KEP, SING); Hervey s.n. (SING 97401, 97380); Holttum 9343 (SING), s.n. (KEP 183949, SING 97388); Hou 768 (KEP); Imin \& Wilkie FRI 68079 (KEP); Kamarudin FRI 28399 (KEP), KMS 1071 (UKMB); Kiew RK 830 (KEP), RK 5089 (KEP, SING), RK 5229 (SING); Koenig s.n. (iso, BM 950666 (http://plants.jstor.org/specimen/bm000950666, last accessed 1 Aug. 2013)); Kunstler 8770 (K); LaFrankie 2092 (KEP); Lake \& Kelsall s.n. (SING, mounted on one sheet with Goodenough 1343); Lee LPB 11 (UKMB); Maingay 1318 (K); Mohd Nur 798 (KEP); Mohd Shah \& Ahmad MS 2321 (SING); Mohd Shah \& Sanusi MS 2167 (SING); Mohzan KEP 98185 (KEP); Ng \& Beltran FRI 6403 (KEP); Nongchie s.n. (SING 97382); Ogata FRI 110415 (K, KEP); Phillips 1644 (KLU); Poore 892 (KLU); Ridley s.n. (SING 97386, 97405); Rogstad 519 (KEP); Sam \& Apok FRI 47145 (KEP); Sam \& Markandan FRI 47029 (KEP); Sani \& Damanhuri SM 348 (UKMB); Santiago \& Lindong 71405 (KEP); Saw FRI 44355 (KEP); Sinclair 8934 (K); Soepadmo ES 403 (KEP); Stone 9468 (KLU), 14631 (KLU), 15067 (KLU); Symington FMS 23341 (KEP), FMS 24403 (KEP); T \& P 650 (SING); W.R.S. s.n. (KLU 15640); Whitmore FRI 12798 (K, KEP, SING); Woods KL 1719 (K, KEP); Yao \& Hughes FRI 65369 (KEP); Yao et al. FRI 65470 (KEP); Yeop 5888 (KEP); Zainudin AZ 2638 (UKMB), AZ 2938 (UKMB); ZAM 41 (UKMB). - SINGAPORE, Burkill HMB 3162 (SING), HMB 3163 (SING); Dawn s.n. (SING 3874); Enoch s.n. (SINU 2007000008); Goodenough s.n. (SING 3883, 3884); Jumali s.n. (SINU 2007000009); Keng 2952 (SINU), s.n. (SINU 2007000011); Kiah s.n. (SING 3887); Lai LJ 177 (SING); Maxwell 78-52 (SINU), 82-27 (KLU, SING); Mohd Nur NK 155 (SING); Ridley 178 (K, SING), 3355 (SING), 4691 (K), s.n. (SING 3876); Saat SING 2005-42 (SING); Samsuri SA 980 (SING); Samsuri et al. NES 131 (SING).

Thottea sumatrana: Peninsular MALAYSIA, Kiah SFN 35290 (K, SING); Rahmat si Toroes 5259 (holo NY 285523 (http://sweetgum.nybg.org/vh/specimen.php?irn=601947, last accessed 29 July 2013), iso US 105817 (http:// collections.mnh.si.edu/search/botany/?ti=3, last accessed 29 July 2013)); Syahida Emiza \& Kueh FRI 57266 (KEP); Yao et al. FRI 65484 (KEP). Thottea tricornis: Peninsular MaLAYSIA: Abdullah KL 248 (KEP); Aminah \& Mala s.n. (UKMB 13855); Anthonysamy SA 339 (KEP); AR \& ZAl s.n. (UKMB 02734); Baharudin BB 44 (UKMB); Burkill \& Mohd Haniff 16765 (SING); Gadoh KL 646 (KEP), KL 713 (KEP); Hamid FMS 10891 (K, KEP, SING); Hamid et al. PUS 179 (UKMB); Hassan \& Kasim HB 099 (UKMB); Hughes et al. FRI 59312 (KEP); Hume 7530 (SING); 9482 (SING); Imin et al. FRI 58645 (KEP), FRI 63114 (KEP); Jamaliah JZED 43 (UKMB); Jamili JN 69 (UKMB); Julius et al. FRI 57736 (KEP); Kamarudin s.n. (UKMB 12058); Kiew RK 1028 (KEP), RK 1238 (SING), RK 1283 (KEP), RK 1542 (KEP), RK 4698 (SING), s.n. (SING 15232); Low 97 (SING); Mohd Hashim FMS 3029 (KEP); Mohd Kasim et al. s.n. (UKMB 01012); Naimah N 46 (UKMB); Ng FRI 6277 (KEP); Provencher Nr T 5, (SING); Putz FRI 21985 (KEP); Razali RS 7 (KEP); Razali \& Rahim RJ 329 (UKMB); Reid s.n. (SING 97414); Sam et al. FRI 47025 (KEP); Saw FRI 40059 (KEP); Syahida Emiza \& Angan FRI 55036 (KEP), FRI 55095 (KEP); Unknown s.n. (SINU 2007000018); Wyatt-Smith KEP 76188 (KEP); Yao FRI 65361 (KEP); Yao \& Kiew FRI 55991 (KEP); Yao et al. FRI 65466 (KEP). 